ARTICLE

\title{
The serine proteases dipeptidyl-peptidase 4 and urokinase are key molecules in human and mouse scar formation
}

Vera Vorstandlechner 1,2,3, Maria Laggner ${ }^{1,2}$, Dragan Copic ${ }^{1,2}$, Katharina Klas ${ }^{1,2}$, Martin Direder ${ }^{1,2}$, Yiyan Chen 4,5, Bahar Golabi ${ }^{4}$, Werner Haslik ${ }^{3}$, Christine Radtke ${ }^{3}$, Erwin Tschachler ${ }^{4}$, Konrad Hötzenecker ${ }^{6}$, Hendrik Jan Ankersmit ${ }^{1,2,7 凶} \&$ Michael Mildner (iD $4,7 凶$

Despite recent advances in understanding skin scarring, mechanisms triggering hypertrophic scar formation are still poorly understood. In the present study, we investigate mature human hypertrophic scars and developing scars in mice at single cell resolution. Compared to normal skin, we find significant differences in gene expression in most cell types present in scar tissue. Fibroblasts show the most prominent alterations in gene expression, displaying a distinct fibrotic signature. By comparing genes upregulated in murine fibroblasts during scar development with genes highly expressed in mature human hypertrophic scars, we identify a group of serine proteases, tentatively involved in scar formation. Two of them, dipeptidylpeptidase 4 (DPP4) and urokinase (PLAU), are further analyzed in functional assays, revealing a role in TGF $\beta 1$-mediated myofibroblast differentiation and over-production of components of the extracellular matrix in vitro. Topical treatment with inhibitors of DPP4 and PLAU during scar formation in vivo shows anti-fibrotic activity and improvement of scar quality, most prominently after application of the PLAU inhibitor BC-11. In this study, we delineate the genetic landscape of hypertrophic scars and present insights into mechanisms involved in hypertrophic scar formation. Our data suggest the use of serine protease inhibitors for the treatment of skin fibrosis.

\footnotetext{
${ }^{1}$ Laboratory for Cardiac and Thoracic Diagnosis, Regeneration and Applied Immunology, Department of Thoracic Surgery, Medical University of Vienna, Vienna, Austria. ${ }^{2}$ Aposcience AG (FN 308089y), Dresdner Straße 87/A21, Vienna, Austria. ${ }^{3}$ Department of Plastic and Reconstructive Surgery, Medical University of Vienna, Vienna, Austria. ${ }^{4}$ Department of Dermatology, Medical University of Vienna, Vienna, Austria. ${ }^{5}$ University of Applied Sciences, FH Campus Wien, Vienna, Austria. ${ }^{6}$ Department of Thoracic Surgery, Medical University of Vienna, Vienna, Austria. ${ }^{7}$ These authors contributed equally: Hendrik Jan Ankersmit, Michael Mildner. ${ }^{凶}$ email: hendrik.ankersmit@meduniwien.ac.at; michael.mildner@meduniwien.ac.at
} 
ypertrophic scars are a complex medical problem and a significant global disease burden ${ }^{1,2}$. In the western world, an estimated number of 100 million people develop scars every year, $\sim 11$ million of which bear keloid scars and 4 million suffer from burn scars ${ }^{1}$. In the USA, an estimated amount of 12 billion dollars is spent annually on the treatment of skin scarring $^{3}$. For affected persons, a pathological hypertrophic scar can cause significant functional impairment, pain, pruritus, and a reduction in quality of life $e^{4,5}$.

Wound healing is a tightly coordinated, three-step process, characterized by an acute inflammatory phase, a proliferative phase, and a remodeling phase. Prolonged inflammation results in increased fibroblast (FB) activity, with enhanced secretion of transforming growth factor beta 1 (TGF $\beta 1$ ), TGF $\beta 2$, insulin-like growth factor (IGF1), and other cytokines ${ }^{6}$. TGF $\beta 1$ drives differentiation of FBs into myofibroblasts, which have a contractile phenotype, are characterized by excessive secretion of ECMcomponents $^{7}$, and are the major contributors to the formation of hypertrophic scars ${ }^{8}$. Mature hypertrophic scars display strong tissue contraction ${ }^{6}$, and dense, parallel, or whorl-like $\mathrm{ECM}^{8}$.

Topical silicone application, compression or massage therapy, intralesional injection of triamcinolone (TAC), corticosteroids, or 5-Fluorouracil (5-FU), laser ablation, and surgery are the most commonly used options for prevention or treatment of hypertrophic scars $6,9-11$. However, many of these therapies lack evidence of efficacy and safety, show high recurrence rates, and mechanisms of actions are still unclear ${ }^{10,12}$.

Recently, several proteases became the focus of drug development in fibrotic diseases, as they have been shown to be involved in ECM-breakdown and the activation of growth factors in tissue remodeling ${ }^{13,14}$. Serine proteases/peptidases constitute a large, diverse group of proteases, divided into 13 clans and 40 families ${ }^{15}$. The group of trypsins comprises proteases contributing to vital processes such as blood coagulation, fibrinolysis, apoptosis, and immunity ${ }^{16}$. Members of this family include urokinase, HTRA1/3 (high-temperature requirement A1/3 peptidase), several coagulation factors and complement components, PRSS-like serine proteases, granzymes, and cathepsin $\mathrm{G}^{16,17}$. Inhibitors of PLAU have been shown to counteract fibrotic processes in cardiac and pulmonary fibrosis in human in vitro studies and in mouse experiments ${ }^{18,19}$. Recently, the serine protease DPP4 became the center of attention, since DPP4 inhibitors (gliptins) have been clinically used for the treatment of diabetes mellitus ${ }^{20}$. DPP4 was also implicated in a variety of fibrotic pathologies, including cardiac, hepatic, renal, and dermal fibrosis $^{21-25}$, and inhibition of DPP4 activity mitigated fibrotic processes in animal models ${ }^{18,19,26-29}$. However, the contribution of serine proteases to human scar formation and the underlying anti-fibrotic mechanisms are so far not known. Even though scRNAseq was previously performed to identify factors important for embryonic ${ }^{30}$ and postnatal ${ }^{31}$ skin development as well as for tissue regeneration ${ }^{32}$ by investigating murine wound healing ${ }^{33}$, scar tissue on single-cell level has not been investigated yet.

Here, we used scRNAseq to thoroughly study gene expression and mechanisms involved in hypertrophic scar formation. We aimed to identify genes regulated in scar tissue, and to uncover potential targets for drug development toward scar-free wound healing or full reversion of a present scar.

\section{Results}

The single-cell landscape of hypertrophic scars. To elucidate the complex biological processes of scar formation, we performed droplet-based single-cell transcriptome analysis of human hypertrophic scar tissue and healthy $\operatorname{skin}^{34}$ (Fig. 1A). In both samples, Unsupervised Uniform Manifold Approximation and
Projection (UMAP)-clustering revealed 21 cell clusters, which were further classified as specific cell types by well-established marker genes (Figure S2A), expression patterns of all clusters (Figure S2B), and transcriptional cluster proximity via a phylogenetic clustertree (Fig. 1B). We identified seven FB clusters, smooth muscle cells and pericytes (SMC/Peri), three clusters of endothelial cells (EC), and lymphatic endothelial cells (LECs), two clusters of $\mathrm{T}$ cells and of dendritic cells (DC), macrophages (Mac), three keratinocyte (KC) clusters, and melanocytes (Mel). All cells of specific subsets were clustered together, and skin and scar samples displayed comparable cellular cluster composition (Fig. 1C, D). Only cluster FB1 was mainly present in scar tissue. The clusters of skin and scars showed different relative cell number ratios (Fig. 1E, F). Whereas FBs represented $40 \%$ of all cells in healthy skin, a significant increase (53\%) was observed in scar tissue. Similarly, we detected more ECs (16.31\%) in scar tissue as compared to normal skin $(8.1 \%)$. Contrary, the relative numbers of epithelial cells (6.37\%) and immune cells $(12.47 \%)$ in mature hypertrophic scars were significantly reduced compared to skin $(22.47 \%$ and $19.97 \%$, respectively).

When comparing scar to skin, we identified considerably more up- (Fig. 1G) than downregulated genes (Fig. 1H), and the most abundant differential gene expression (number of differentially expressed genes, $\mathrm{nDEG}$ ) was found in $\mathrm{FBs}$, SMC/PCs, macrophages, DC1 and KC1 (Fig. 1G, H). The top 50 up- and downregulated genes for FBs, SMC/PCs, ECs, T cells, DCs, and $\mathrm{KCs}$ are listed in Figure S3. Genes related to ECM production (e.g., COL1A1/2, COL3A1, COL5A1/2, FN1, BGN, LOX, LUM, OGN, PCOLCE) were mainly overrepresented in FBs, but notably also in PCs and ECs (Figure S3A-C). Several significantly regulated genes with so far undescribed roles in fibrosis and scar formation (e.g., $A R L 4 C, C O P Z 2, C R A B P 2, H S P A 1 A / B, M D K$, $O G N$, among others) were found in all cell types (Figure S3A-F). These distinctly regulated genes might provide valuable new candidates to understand and modulate skin scarring.

The fibrotic gene expression pattern of fibroblasts in hypertrophic scars. Since FBs showed the strongest gene regulation in our scRNAseq dataset, and have been considered as the major drivers of skin scarring and an important source for myofibroblasts ${ }^{7}$, we focused our further analysis on differences between FBs of healthy skin and hypertrophic scars (Fig. 2).

After subsetting and reclustering of all FBs, we identified 11 separate clusters (Fig. 2A-C) showing 110 significantly upand 85 downregulated genes in FBs derived from scar tissue compared to healthy skin. The top 50 differentially up- and downregulated genes are shown in Fig. 2D. Interestingly, one FB cluster (FB1) was almost exclusively present in hypertrophic scars, suggesting a specific role in tissue fibrosis. Comparison of FB cluster 1 to all other scar FBs revealed 141 significantly upand 179 downregulated genes. The top 50 differentially up- and downregulated genes are shown in Fig. 2E. Most of the upregulated genes in scar-derived FBs are well-studied in the context of skin scarring and are functionally related to collagens and ECM-modifying genes, e.g., BGN, COL14A1, COL1A1/2, COL3A1, COL5A1/2, FN1, MMP23B, OGN, PCOLCE (Figure S3). Analysis of the biological processes associated with differentially regulated genes between FB1 and other FB clusters by gene ontology network analysis revealed a strong association of FB1 with TGF $\beta$-signaling (associated genes: ASPN, COL1A1, COL1A2, FBN1, HSPA1A, HTRA1, INHBA, JUN, LOX, POSTN, red circles) and ECM-formation (associated genes: $A E B P 1, B G N, C C N 2$, COL12A1, COL14A1, COL1A1, COL1A2, COL3A1, COL5A1, COL5A2, COL6A1, COL6A2, COMP, CREB3L1, DPP4, EGFL6, FBN1, FN1, HTRA1, LOX, LUM, MFAP2, MMP11, PHLDB2, 
(A)

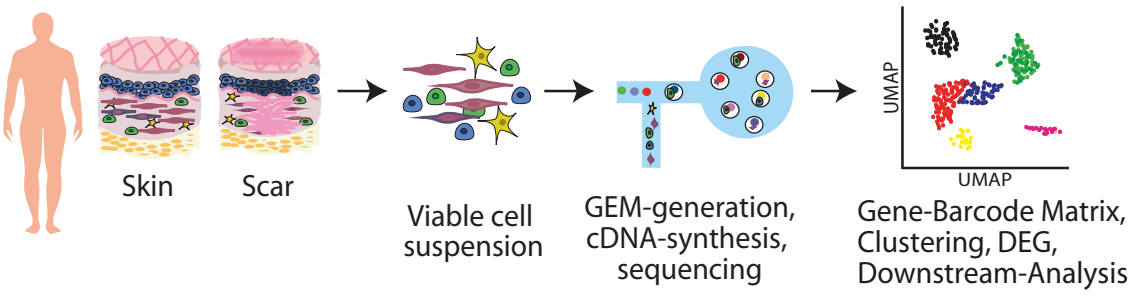

(B)
(C) Skin
(D)
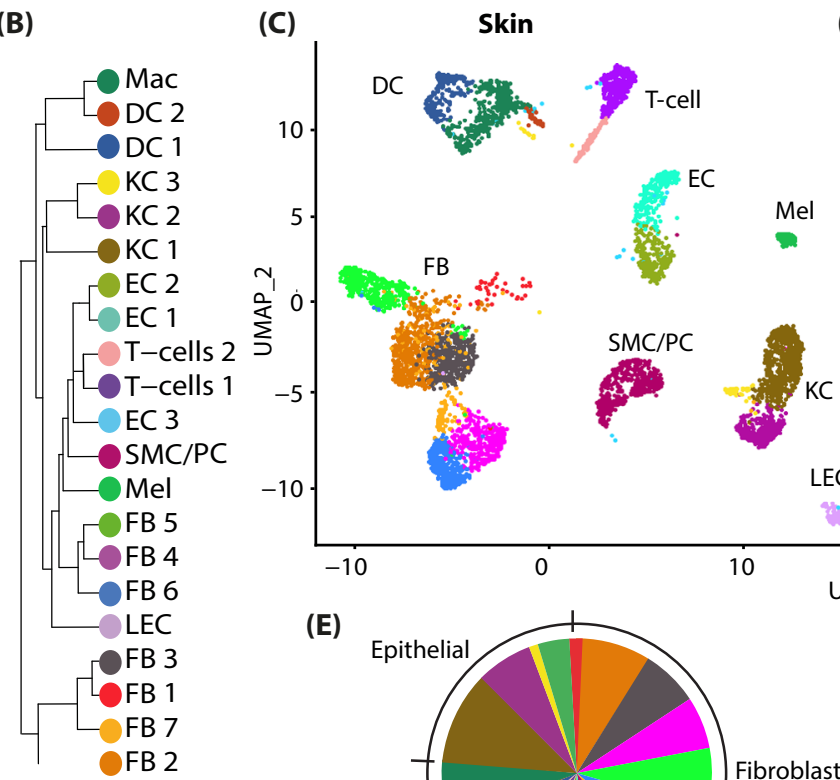

(D)

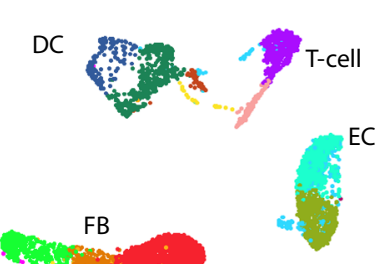

Mel

(E)

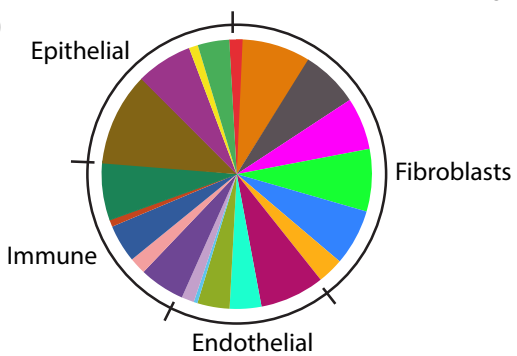

(F)
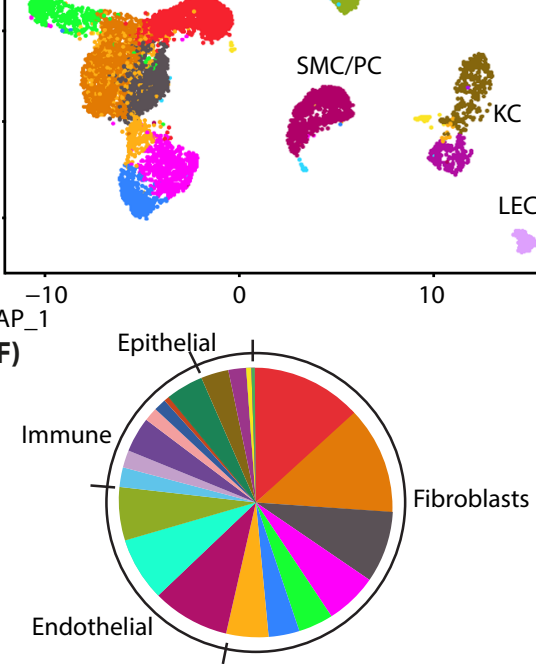

LEC

(H)
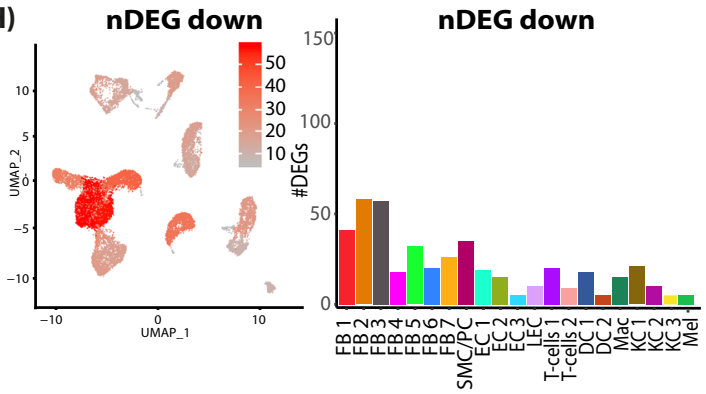

Fig. 1 Characterization of human skin and scar samples with scRNAseq identifies specific cell clusters and a distinct fibrotic gene signature.

A Workflow of scRNAseq in human skin $(n=3)$ and scar $(n=3)$ samples. B Phylogenetic clustertree calculated based on UMAP-clustering. C, D UMAP-

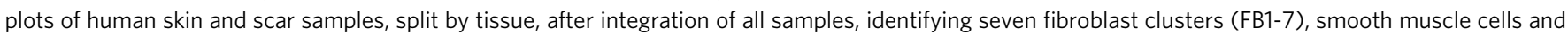
pericytes (SMC/Peri), endothelial cells (EC1 +2$)$, lymphatic endothelial cells (LEC), T cells, macrophages (Mac), dendritic cells (DC1 + 2), three keratinocyte clusters (KC1-3), and melanocytes (Mel). E, F Pie charts showing ratios of cell clusters in skin and scars. Feature plots and bar graphs of number of differentially expressed genes (nDEG) per cluster of $\mathbf{G}$ up- and $\mathbf{H}$ downregulated genes. DEGs were calculated per cluster comparing scar versus skin using Wilcoxon rank-sum test, including genes with average logarithmic fold change (avglogFC) of $>0.1$ or $<-0.1$ and Bonferroni-adjusted $p$-value $<0.05$. Feature plots show projection of nDEG onto the UMAP-plot, color intensity represents nDEG. Bar graphs show absolute numbers of nDEG per cluster, $y$-axis represents nDEG. UMAP, uniform manifold approximation and projection.

POSTN, SERPINE1, SFRP2, SPARC, TGFBI, THBS1, TNC, $V C A N$, purple circles) (Fig. $2 \mathrm{~F}$ ), further corroborating its role in skin fibrosis. In addition, our analysis indicated a role of FB1 in processes important for several other cell types, including platelets, smooth muscle cells (associated genes: CCN3, CHN1, IGF1, IGFBP3, PLAT, PLAU, POSTN, SERPINE1, green circles), and cells of the skeletal system (associated genes: $C C N 2, C C N 3$, COL12A1, COL14A1, COL1A1, COL1A2, COL3A1, COL5A2, COL6A1, COL6A2, COMP, ECM1, FBN1, FRZB, HYAL2, IGF1,
INHBA, LOX, LUM, PAPSS2, POSTN, SFRP2, SFRP4, SOX4, SPARC, TGFBI, VCAN, yellow circles), suggesting paracrine actions of FB1.

Pseudotime calculation and trajectory construction effectively identified possible cell fates and time-regulated genes, even when analyzing cells of only one timepoint ${ }^{35,36}$. Thus, we next sorted human skin and scar FBs along a pseudotime axis and constructed trajectories (Fig. 3A, B). The trajectories revealed a division at a certain timepoint where FBs divided into two 
(A)

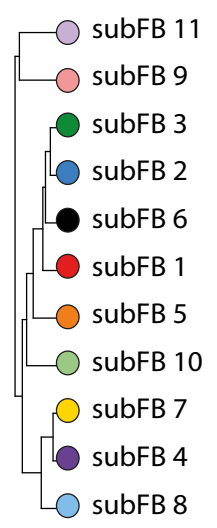

(B) Skin

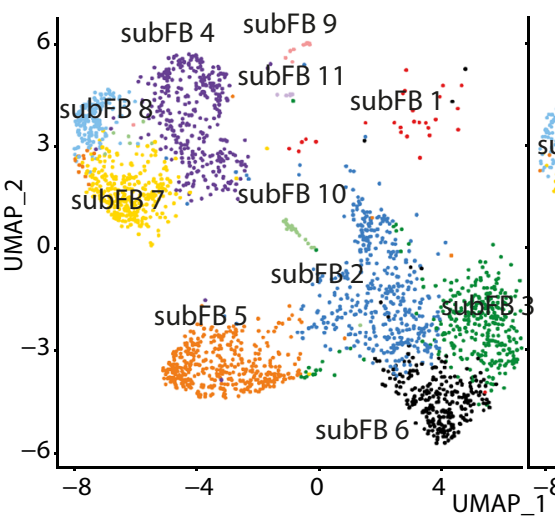

(C)

Scar
(D)
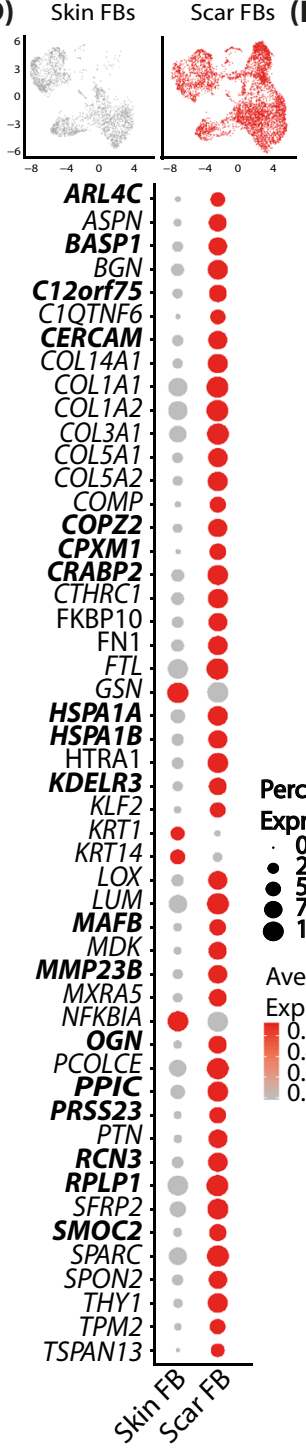

(E) Scar rest FBs Scar subFB1

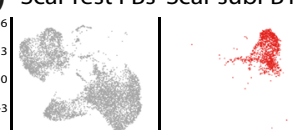

(F)
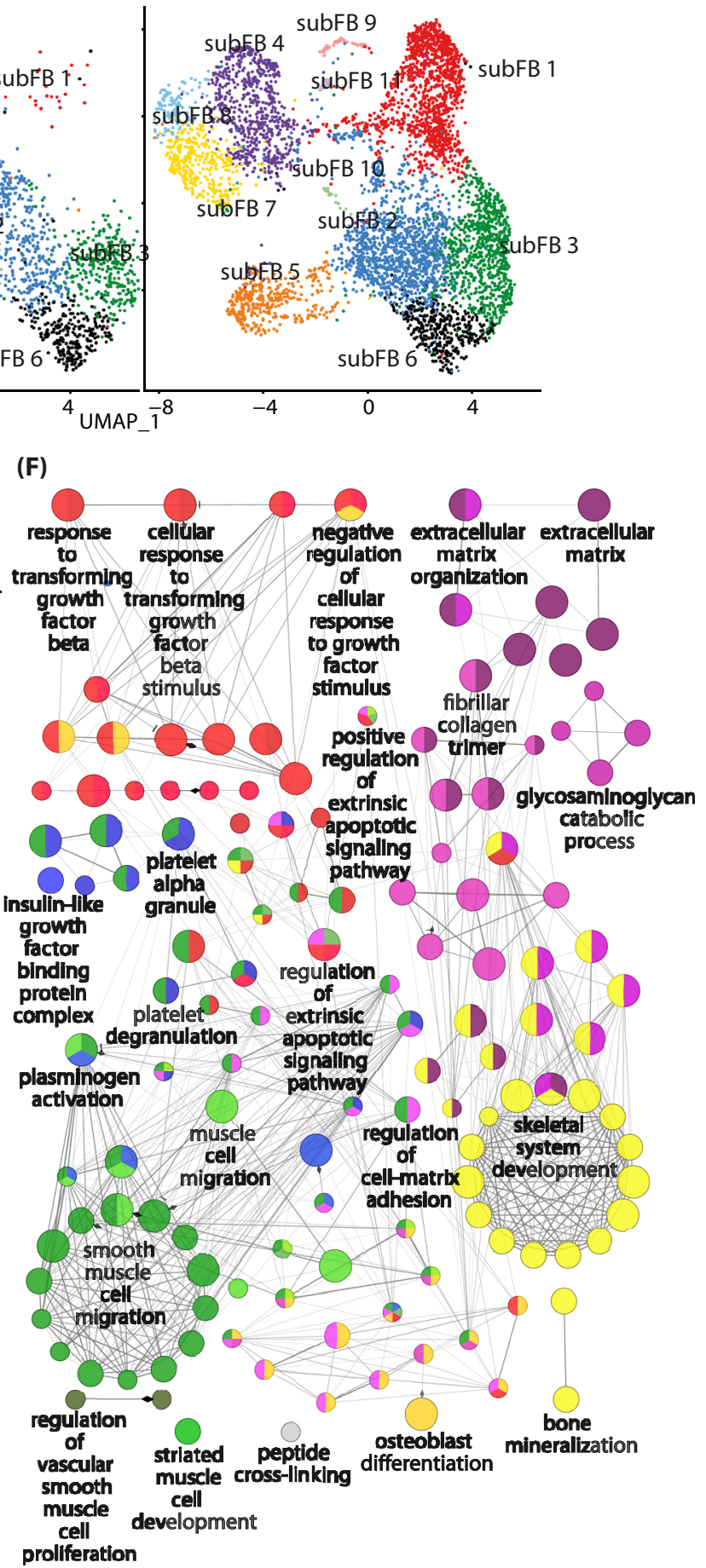

prollferation

branches (Fig. 3C). Whereas the majority of FBs preferentially aligned with branch 1 in normal skin (Fig. 3D), we observed a significantly longer branch 2 with FBs of hypertrophic scar tissue (Fig. 3E). Branched expression analysis identified genes most regulated in a pseudotime-dependent manner in normal skin and hypertrophic scars (Fig. 3F). Interestingly, the collagens COL1A1, COL1A2, and COL3A1, known to contribute to all fibrotic processes, are most upregulated at the end of Branch 2, but are not among the most pseudotime-regulated genes in scar (Fig. $3 \mathrm{~F}$ ). In contrast, other collagens, including COL5A1/2, COL8A1, COL11A1, and COL12A1, dominated the late pseudotimedependent gene expression in branch 2. The role of these collagens in (hypertrophic) scar is scarcely investigated, and merits further exploration. Together, our trajectory analysis 
Fig. 2 Analysis of fibroblast subsets. A Phylogenetic clustertree calculated based on UMAP-clustering of subsetted fibroblasts only. B, C UMAP-plots of re-clustered skin and scar fibroblasts, split by tissue, reclustering identified 11 fibroblast clusters (subFB1-11). D Feature plots illustrating computational basis for dotplots. Dotplots of top 50 regulated genes (according to lowest adjusted p-value) comparing scar FBs versus skin FBs. E Dotplot of top 50 regulated genes (according to lowest adjusted $p$-value) cluster subFB1 compared to all other scar FBs. F Gene ontology-term network was calculated based on significantly upregulated (adj. $p$-value $<0.05$, avg.logFC $>0.1$ ) genes comparing subFB1 to all other scar FBs. Gene list was imputed in ClueGO plug-in in Cytoscape with medium GO-specificity, with GO-term fusion, only significant ( $P$ value $<0.05)$ GO terms are shown. Circle size correlates with $P$ value, lines ("edges") represent functional connection of respective GO terms. Red circles represent association of GO-term with TGF $\beta$-signaling, purple, with extracellular matrix, green, with smooth muscle differentiation, blue, with signaling factors, and yellow with bone formation and -development. UMAP, uniform manifold approximation and projection.

models the temporal dynamics of gene expression in scars and might provide a basis to target respective genes at different stages of scar development. Interestingly, no genes were strongly regulated directly at the branching point, suggesting cell-fate is already determined at the beginning of pseudotime.

scRNAseq of murine scars identifies genes involved in scar maturation. As our approach so far only gave information on the current state of mature scars, we further investigated mechanisms leading to scar formation and maturation, using a murine fullthickness skin wound model (Figure S4A). Whereas scar formation and maturation in humans is a long-lasting process ${ }^{37}$, it only takes up to 80 days in rodents ${ }^{38}$. Although the here used murine scar model does not completely reflect hypertrophic scar formation in humans, the analysis of genes that are regulated in both, human hypertrophic scars and during normal scar formation in mice, might identify the most evolutionary conserved and most interesting targets for therapeutic interventions.

In order to detect dynamic differences in gene expression related to scar formation rather than wound healing, we compared samples from normal mouse skin, and from mice 6 and 8 weeks after wounding (Fig. 4A). Analogously to the human dataset, the murine scRNAseq dataset was clustered, and cell types were identified using established marker genes (Figure S4B), expression patterns of all clusters (Figure S4C), and transcriptome proximity of clusters via a phylogenetic clustertree (Fig. 4B). All clusters aligned homogeneously, and all major skin cell types were represented in normal skin and at both time points after scar development (Fig. 4C, Figure S4). In accordance with human scar tissue, 8 -week-old mouse scars contained a higher proportion of murine FBs (mFBs) (32.6\%) compared to 6-week-old scars (17.39\%), and more immune cells (9.6 versus $6.3 \%)$. In contrast, less of the endothelium (2.8 versus $1.5 \%$ ) and less keratinocytes (63.3 vs $45 \%)$ were present (Fig. $4 \mathrm{D})$. We next calculated up- and downregulated genes for FBs, PCs, ECs, T cells, DCs, and KCs, comparing 8 weeks to 6-week-old scars (top 50 are shown in Figure S5A-F). In contrast to human scars, the highest number of differentially expressed genes was found in mFBs and mKCs (Fig. 4E, F), which was most likely due to ongoing epidermal tissue regeneration. Expression of Acta2 and collagens showed only minor regulation between 6 and 8 weeks in mFBs (Fig. 4G). In addition, expression of several other matricellular and ECMmodulating proteins, e.g., Fbln1 (Fibulin1), Ogn (osteoglycin), Lum (Lumican), and Pcolce (Procollagen C-Endopeptidase Enhancer), and Tgfbi (transforming growth factor, beta-induced) increased in mFBs during scar maturation (Fig. 4H). Together, our scRNAseq identified a gene profile specific for scar maturation in mice.

Serine proteases are strongly upregulated during scar maturation. To identify genes that are crucial for scar maturation, we next compared our human scar dataset with genes upregulated in mouse scars 8 weeks after wounding in comparison to mouse scars 6 weeks after wounding (Fig. 5A). While in both datasets only one gene (LEPR) was downregulated, 16 genes were mutually upregulated (Fig. 5B-D). Stunningly, 5 of these genes (AEBP1,DPP4, HTRA1, PLAU, and PRSS23) were members of the superfamily of serine proteases (Fig. 5C, E). All five serine proteases were upregulated in scRNAseq in human scar tissue, particularly in FBs, but also in other cell types (Fig. 5E-J). AEBP1 and PRSS23 expression also increased in ECs and melanocytes, HTRA1 in ECs and KC3, and PLAU in DCs. Several additional serine proteases, HTRA3 (high-temperature requirement A serine peptidase 3), DPP7 (dipeptidyl-peptidase 7), FAP (fibroblast activation protein alpha), were upregulated in human scars (Figure S6), and also showed a trend in mouse scars. Analysis of theses serine proteases by pseudotime trajectories in human FBs revealed that their expression mainly increased over time and $A E B P 1$ and HTRA1 significantly enriched at the end of branch 2 (Figure S7). Together, these data suggest a major role of serine proteases in scar formation and/or maturation.

The serine proteases DPP4 and urokinase regulate TGF $\beta 1$ mediated myofibroblasts differentiation and ECM overproduction. We next wanted to investigate the contribution of the identified serine proteases to scar formation. Since specific inhibitors are commercially available only for DPP4 and urokinase, we focused our further functional studies on these two serine proteases. First, we corroborated our scRNAseq data by analyzing RNA and protein expression of DPP4 and urokinase (PLAU) using in situ hybridization (Figure S8), and immunofluorescence staining of human (Fig. 6A-C) and murine (Figure S8E-G) skin and scars. Immunofluorescence staining revealed expression of urokinase in the dermis and epidermis of healthy skin. In contrast, DPP4 was only present in the dermal compartment of healthy skin. Whereas the expression of DPP4 was significantly increased in the epidermis and dermis of hypertrophic scars in both species, immunofluorescence staining revealed only a slight, not significant upregulation of PLAU in the dermal compartment of hypertrophic scars. Since detection of released proteins by immunofluorescence often shows difficulties, we further quantified urokinase and DPP4 in human tissue biopsies using ELISA. Interestingly, both urokinase and DPP4 were significantly increased in human scar tissue compared to normal skin (Fig. 6F, G).

As TGF $\beta 1$ is one of the key inducers of scarring and tissue fibrosis, causing differentiation of FBs to profibrotic myofibroblasts $19,39-41$, we hypothesized that the serine proteases interact with TGF $\beta$-signaling. To test this, we performed siRNAmediated gene knockdown of DPP4 and PLAU in primary FBs from healthy human skin. The knockdown significantly downregulated DPP4 and PLAU mRNA expression levels (Figure S9A, $B)$ and almost completely abolished the production of the respective proteins (Fig. 7A). Knockdown of both genes strongly reduced TGF $\beta 1$-mediated expression of alpha-smooth muscle actin (aSMA), a marker for myofibroblasts (Fig. 7B). The reduced aSMA expression was accompanied by a reduced ability to 


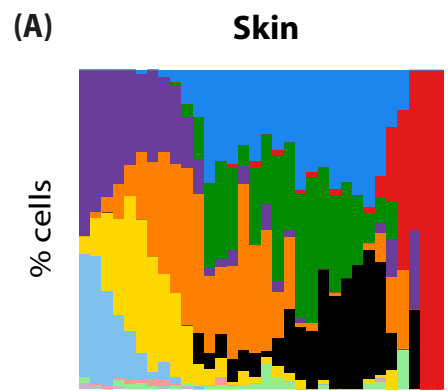

Pseudotime
(B) Scar

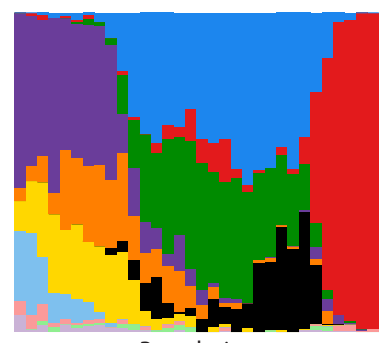

Pseudotime

(C)
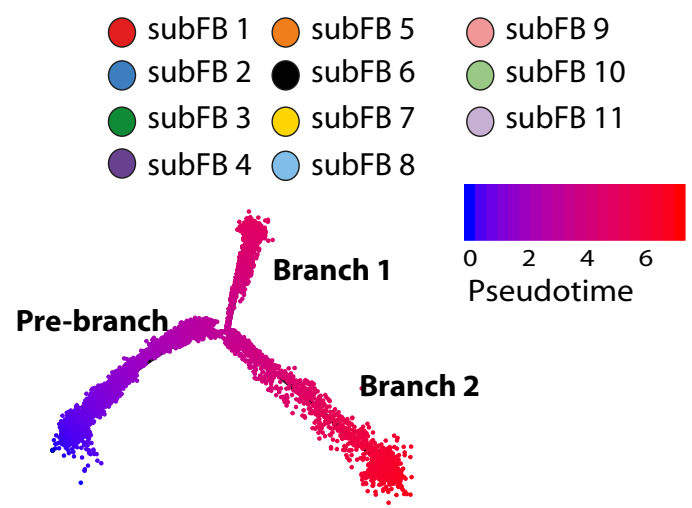

(D)

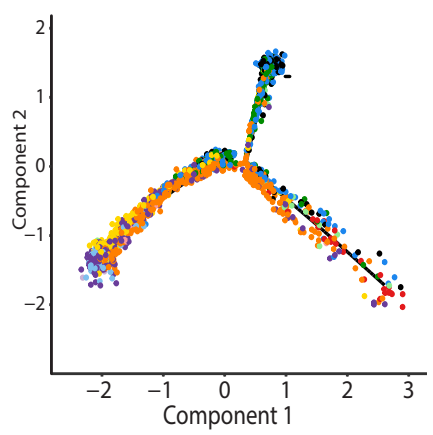

(E)

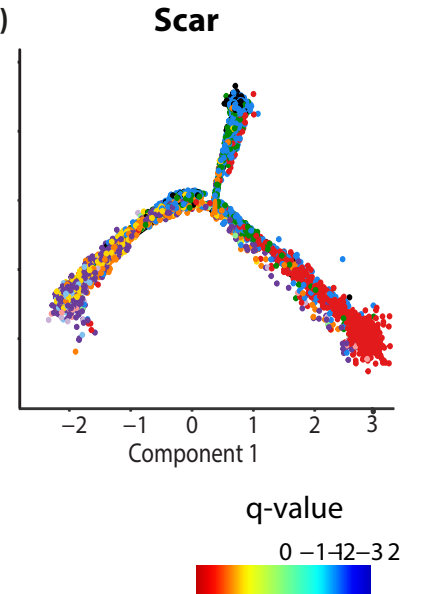

(F)
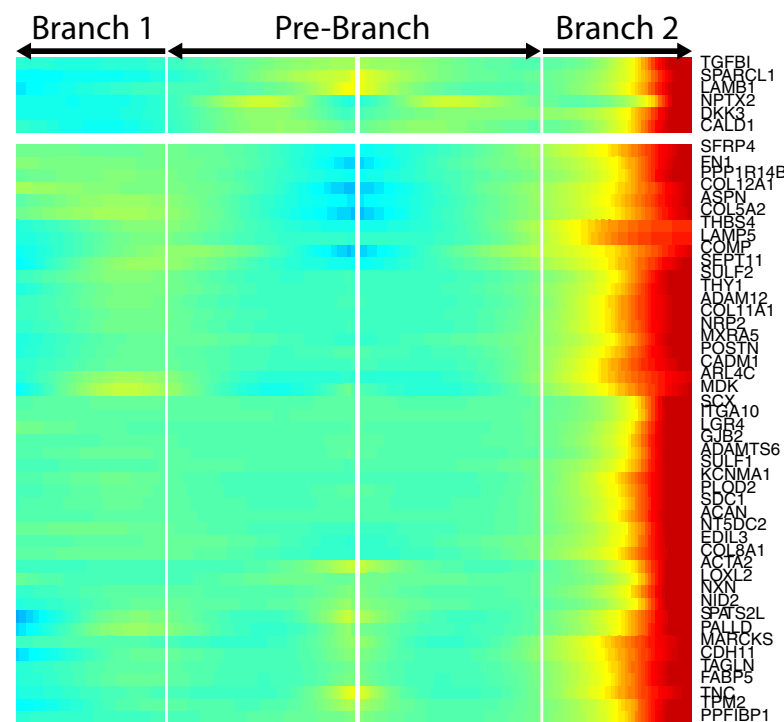

SFRP4

SFRP4

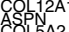

ASPA2
CABS
LAMP5
LAMP

STup 21

ADAM12

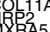

CADM1

MDK

SEX 10

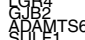

SCKFMA

SLOC 12

ACAN

COLBA

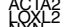

tox 2

NND
NDAS2L
PALLD
PLLCK

PALLD

TAALN

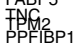

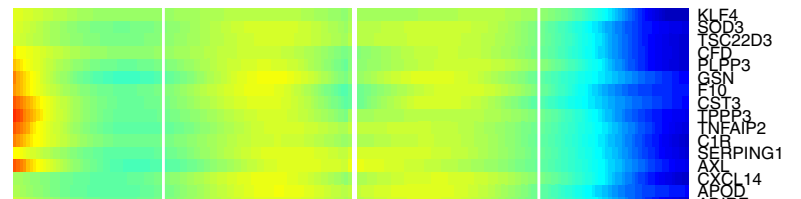

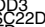

NEAIP2

Ab

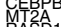

能穴1

竪 362

CSS 1 .
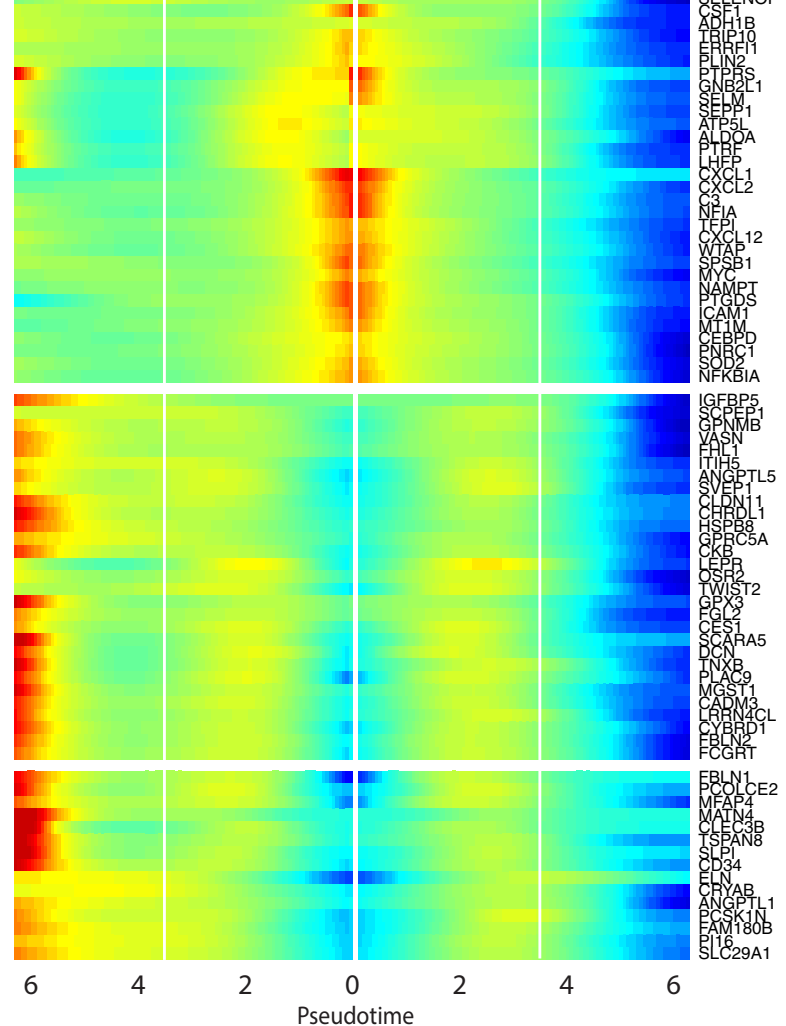

Pseudotime

Fig. 3 Pseudotime analysis of human scar FBs identifies cell fates and pseudotime-regulated genes. A, B Ordering skin and scar FBs along a pseudotime axis. $X$-axis, pseudotime. $y$ axis, \% of cells in respective pseudotime-bin. Cell trajectory with pre-branch and branches is shown. C Color code represents pseudotime progression. D, E Cell trajectories were calculated based on pseudotime values, split by tissue. $\mathbf{F}$ Branched expression analysis modeling (BEAM) of skin and scar fibroblasts. Colors represent $q$-value, the expression of the respective gene in relation to pseudotime. 
(A)

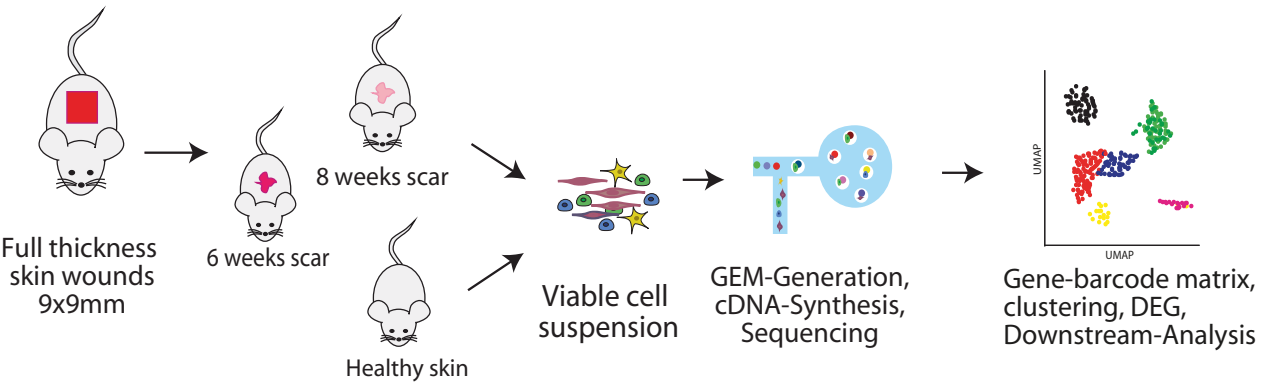

(B)

(C)

Skin
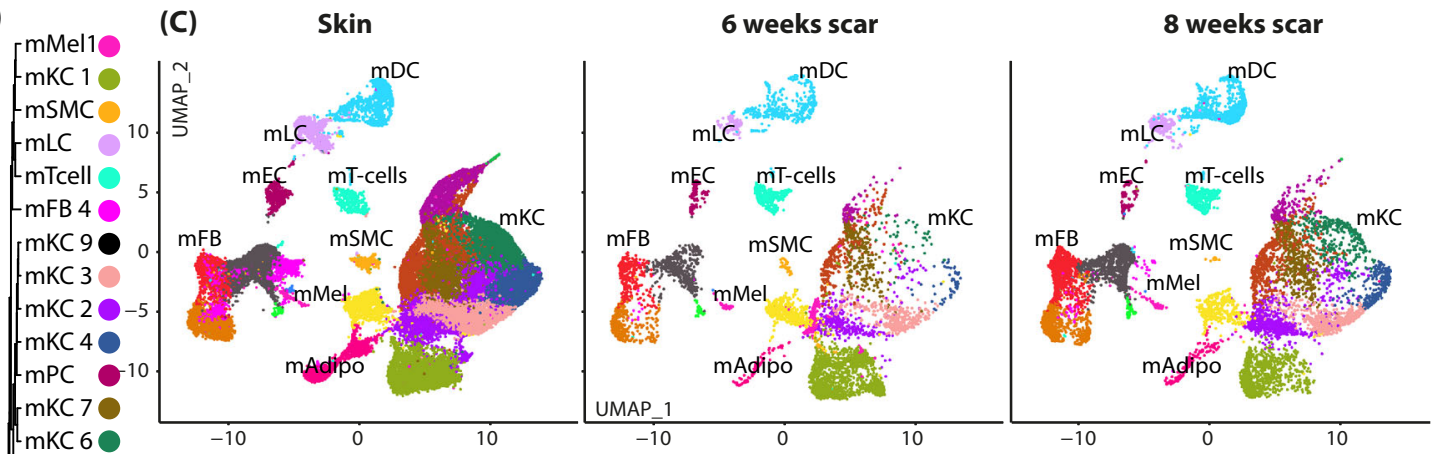

mKC 60

mFB $3 \bigcirc$ (D)
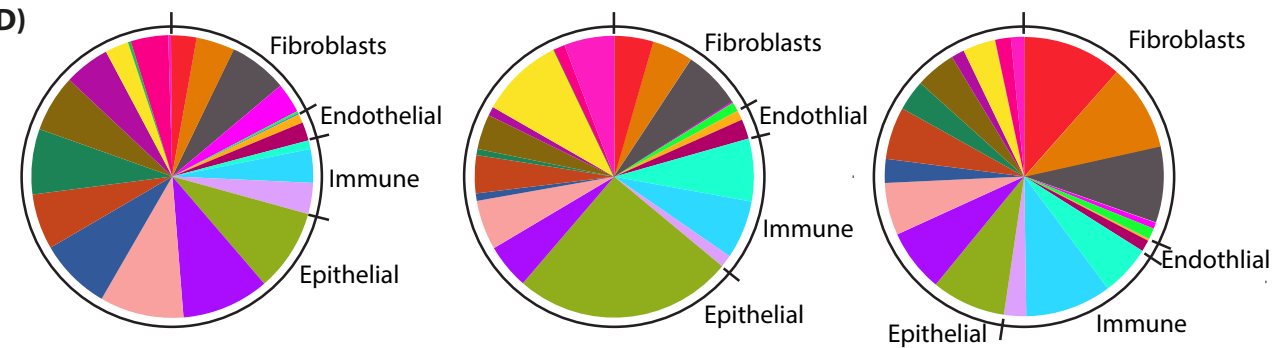

(E)
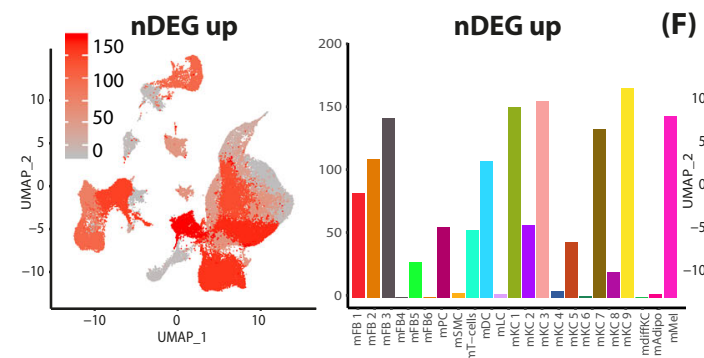

(F) $\mathrm{nDEG}$ down

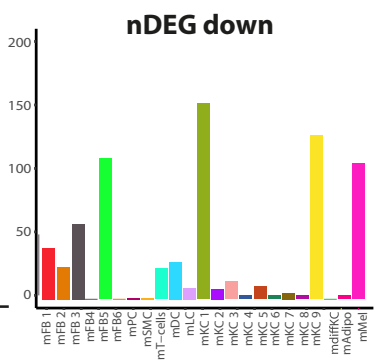

(G)

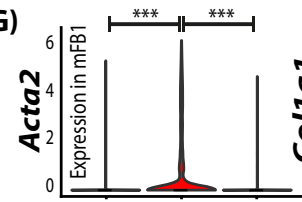

(H)
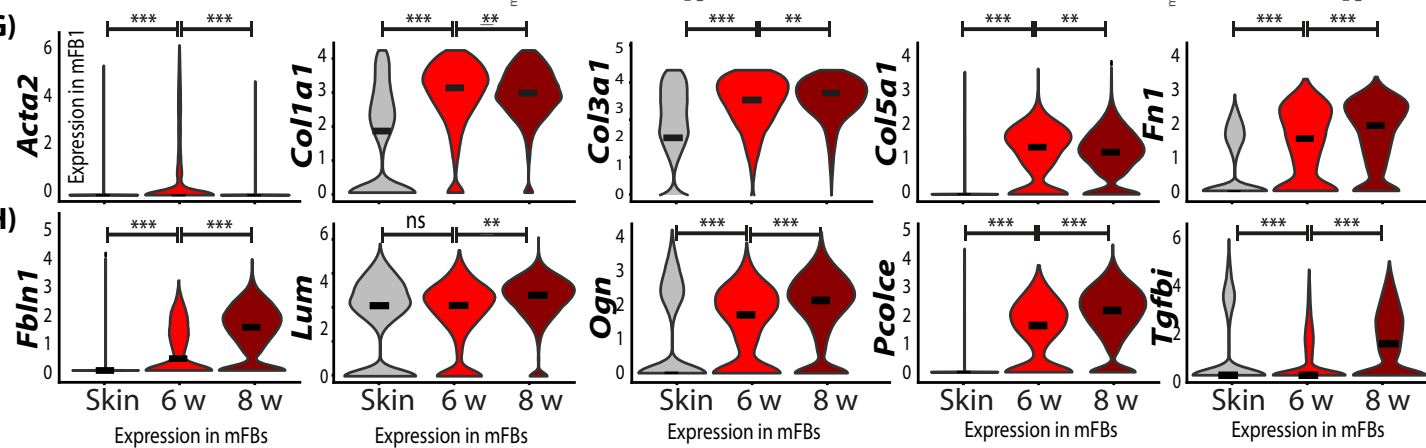

contract a matrix of collagen in vitro (Fig. 7C). We also analyzed components of the ECM and found significantly lower expression of different collagens and fibronectin (FN1) in knockdown FBs (Figure S9C-F). While FN1 protein release was strongly reduced (Fig. 7E), protein levels of COL1al were not reduced $48 \mathrm{~h}$ after gene silencing (Fig. 7D). Of note, transfection of cells led to a strong increase of baseline levels of FN1 and
COL1al, which might be the reason for the weak response to TGF $\beta 1$ stimulation.

Next, we assessed these effects using the specific inhibitors for DPP4 (Sitagliptin) and PLAU (BC-11). Both inhibitors were able to abolish TGF $\beta 1$-induced $\alpha \mathrm{SMA}$ production to a comparable degree as the specific gene knockdown (Fig. 7F, G). Surprisingly, collagen contraction was not inhibited with the inhibitors 
Fig. 4 Two-timepoint mouse scar model identifies genes regulated in scar maturation. A Workflow of mouse skin scar model and two-timepoint $(n=2$ per timepoint) scRNAseq. B Phylogenetic clustertree calculated unsupervised based on unsupervised UMAP-clustering. C UMAP-plots of mouse scar tissue, split by timepoint, after integration of all samples, identifying four fibroblast clusters (mFB1-4), smooth muscle cells and pericytes (mPC/SMC), endothelial cells and lymphatic endothelial cells (mEC/LEC), T cells, dendritic cells (mDC), Langerhans cells (mLC), nine keratinocyte clusters (KC1-9), adipocytes (mAdipo), and melanocytes (Mel). D Pie charts show relative numbers of cells in clusters, split by timepoint. Feature plots and bar graphs of number of differentially expressed genes (nDEG) per cluster of $\mathbf{E}$ up- and $\mathbf{F}$ downregulated genes per cluster. $\mathbf{G}, \mathbf{H}$ Violin plots of ECM-associated genes. Acta2 skin vs $6 \mathrm{w} p=2.22 \mathrm{e}-16 ; 6 \mathrm{w}$ vs $8 \mathrm{w} p=1.4 \mathrm{e}-6$; Colla1 $p=2.22 \mathrm{e}-16, p=0.23 ;$ Col3a1 $p=2.22 \mathrm{e}-16, p=0.0079$, Col5a1 $p=2.22 \mathrm{e}-16 ; p=5 \mathrm{e}-5$, Fn 1 $p=2.22 \mathrm{e}-16 ; p=5.5 \mathrm{e}-10 ;$ Fbln1 $p=1.3 \mathrm{e}-10,2.22 \mathrm{e}-16 ;$ Lum $p=0.065, p=2.22 \mathrm{e}-16 ;$ Ogn $p=9.3-0.5, p=2.22 \mathrm{e}-16 ;$; Polce $p=2.22 \mathrm{e}-16,2.22 \mathrm{e}-16$; Tgfbi $p=0.023, p=2.22 \mathrm{e}-16$. Vertical lines in violin plots represent maximum expression, shape of each violin represents all results, and width of each violin represents frequency of cells at the respective expression level. DEGs were calculated per cluster comparing 8-vs 6-week-old scars using a twosided Wilcoxon-signed rank test, including genes with average logarithmic fold change (avg_logFC) of $>0.1$ or $<-0.1$ and Bonferroni-adjusted $p$-value $<0.05$. Feature plots show projection of nDEG onto the UMAP-plot, color intensity represents nDEG. Bar graphs show absolute number of nDEG per cluster, $y$-axis represents nDEG. UMAP uniform manifold approximation and projection.

(Fig. 7H), indicating off-target or unspecific inhibitor effects. Moreover, Sitagliptin and BC-11 attenuated TGF $\beta 1$-induced overproduction of the ECM-proteins Collal (Fig. 7I), and fibronectin (Fig. 7J) by FBs. These results demonstrate that serine proteases are involved in TGF $\beta 1$-induced myofibroblast differentiation. Of note, not all observed effects found in FBs deficient for PLAU or DPP4, were mirrored with pharmacological inhibitors.

To investigate whether the serine protease inhibitors interfere with TGF $\beta 1$ signaling, we analyzed the TGF $\beta 1$-induced SMAD and ERK signaling pathways ${ }^{42}$. Neither knockdown of DPP4 or PLAU nor addition of the inhibitors led to a significant inhibition of the SMAD and ERK1/2 signaling pathway (Figure S10A). To further identify other signaling pathways that might be involved in the action of the serine protease inhibitors, we used a signaling proteome profiler, showing that none of the signaling molecules were blocked by the inhibitors (Figure S10B). Interestingly, the GSKa/ $\beta$-pathway, known to attenuate fibrotic processes in the heart $^{43}$ was significantly activated by BC-11 (Figure S10B-D), indicating a counter-regulatory action. Together, these data suggest that sitagliptin and BC-11 do not interfere with canonical or known non-canonical TGF $\beta 1$ signaling.

The serine protease inhibitors Sitagliptin and BC-11 improve scar formation by interfering with production and organization of the ECM. We next attempted to assess the effects of Sitagliptin and BC-11 in in vivo scar formation in mice (Fig. 8A). Application of the inhibitors did not interfere with wound healing (Fig. 8B), and even showed a slight, non-significant trend toward faster wound closure after application of BC-11 (Fig. 8C). scRNAseq of scars (Fig. 8D-J) showed a lower number of the main matrix producing $\mathrm{FB}$ clusters $\mathrm{mFB} 1$ and $\mathrm{mFB} 2$ in $\mathrm{BC}-11$ stimulated scars after 8 weeks (Fig. $8 \mathrm{~F}, \mathrm{I}$ ). The top 50 regulated genes are shown in Figure S11. Treatment of mice scars with BC11 and Sitagliptin resulted in a slightly higher expression of Col1a1, but significantly lower expression of Col3a1, Col5a1, and Fn1. Interestingly, both inhibitors reduced the expression of their target proteases. Of note, $\mathrm{BC}-11$ treatment also strongly reduced Dpp4 expression (Fig. 8K).

To assess formation of the ECM and collagen deposition, we stained skin and scar samples with picrosirius red (Fig. 9A) and with antibodies against collagen 3 (Fig. 9B), and fibronectin (Fig. 9C). Sirius red staining showed a reduction in total collagen deposition after treatment with both inhibitors (Fig. 9A). Immunofluorescence stainings revealed a significant alteration in collagen alignment and size of the collagen bundles between skin and scars but also between untreated scars and scars treated with inhibitors. As shown in Fig. $9 \mathrm{~B}$ and $\mathrm{C}$, both inhibitors strongly reduced the thickness of collagen bundles. To assess the quality of the resulting scar tissue, we used CurveAlign, a tool designed to measure orientation of the ECM. Comparable areas directly adjacent and parallel to the epidermis were analyzed in H\&E-stained sections of skin and scars (Fig. 9B). An alignment coefficient was calculated from orientation and alignment of collagenous fibers. A lower coefficient indicated less parallelism and thus less dense dermis. Strikingly, BC-11 treated scars showed a significantly lower alignment coefficient than control scars (Fig. 9C). This effect was not observed in sitagliptin-treated scars. Together, our data suggest that Sitagliptin, and even more prominently BC-11 interfere with matrix deposition in vivo, representing promising candidates for the improvement of (hypertrophic) skin scar formation.

\section{Discussion}

Although skin fibrosis has been extensively studied, key mechanisms leading to the development of hypertrophic scars are still not well understood. In addition, treatment options to prevent or treat (hypertrophic) scars are still scarce ${ }^{44}$ and not exceptionally effective. In the present study, we used scRNAseq to elucidate the genetic landscape of hypertrophic scar tissue at a hitherto unmet single-cell resolution.

As expected, our scRNAseq analysis confirmed a plethora of previous studies, but also identified numerous genes, which have so far not been described in the context of skin scarring or tissue fibrosis. For example, the cytokines $M D K$ (midkine) and PTN (pleiotrophin), both involved in cell growth, migration, and angiogenesis ${ }^{45}$, were strongly upregulated in scar FBs. In contrast, SOD2/3 (superoxide dismutase 2/3), an enzyme controlling the release of reactive oxygen species (ROS), hence acting as important antioxidant ${ }^{46}$, was strongly downregulated in scar FBs. Intriguingly, failure of ROS-scavenging has already been shown to contribute to hypertrophic scar formation ${ }^{47}$. Another interesting and significantly downregulated gene in scars was SFN (stratifin). As stratifin has been identified as potent collagenase-stimulating factor in FBs, its downregulation in scars suggests a contribution to the maintenance and/or progression of the fibrotic phenotype by preventing matrix degradation. However, we also identified interesting, so far undescribed differences in other cell types. In human SMC/Pericytes for example, we found a strong upregulation of a group of methallothionins (MT1G, MT1E, MT2A, $M T 1 A$ ), which were previously found to be increased in keloid FBs and concomitantly regulated with collagens upon treatment with $\mathrm{TGF} \beta^{48}$, however, their role in hypertrophic scars has yet to be determined. We also identified a rearrangement of $\mathrm{T}$-cell subsets in mouse scar tissue (Figure S12). In the light of a previous publication by Kalekar et al. ${ }^{49}$, demonstrating that GATA3expressing regulatory $\mathrm{T}$ cells contribute to $\mathrm{FB}$ activation in murine dermal fibrosis, and our finding, that Gata3 is strongly upregulated in mouse scars, it is likely that T-cell subsets contribute significantly to scar formation. However, we were not able 
(A)

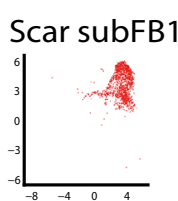

Scar rest FB

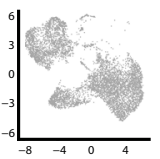

(B)

subFB1 vs rest scarFB

(131)

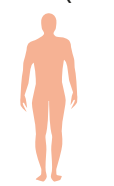

subFB1 vs rest scarFB $\quad \mathrm{mFB} 8 \mathrm{w}$ vs $\mathrm{mFB} 6 \mathrm{w}$

(179)

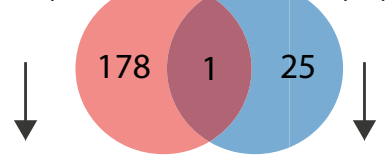

(C)

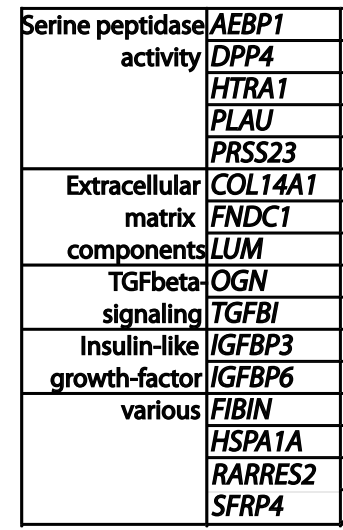

(E)

(F)
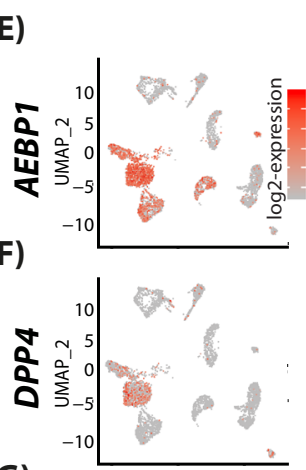

(G)

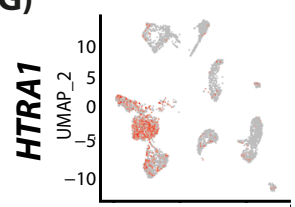

(H)

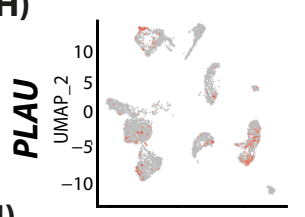

(I)

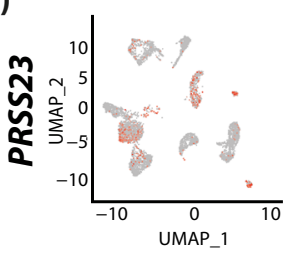

Skin
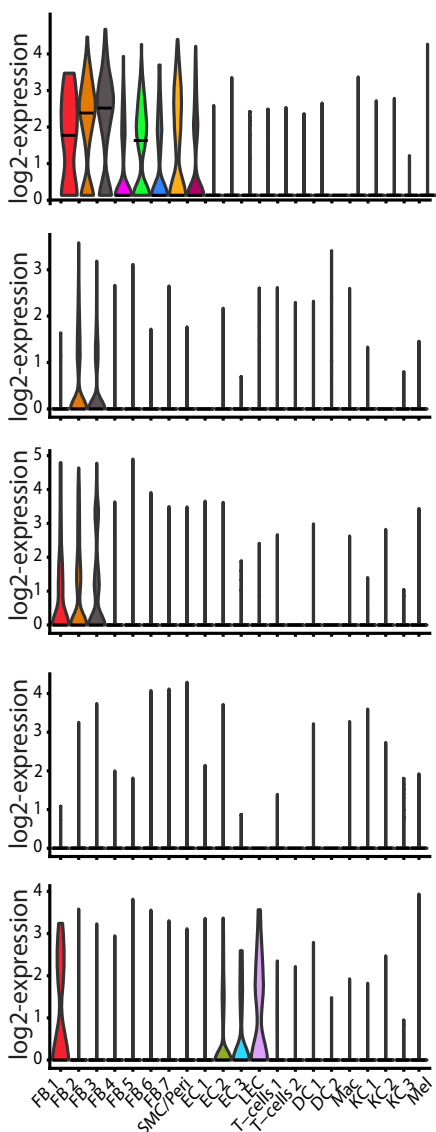

Scar
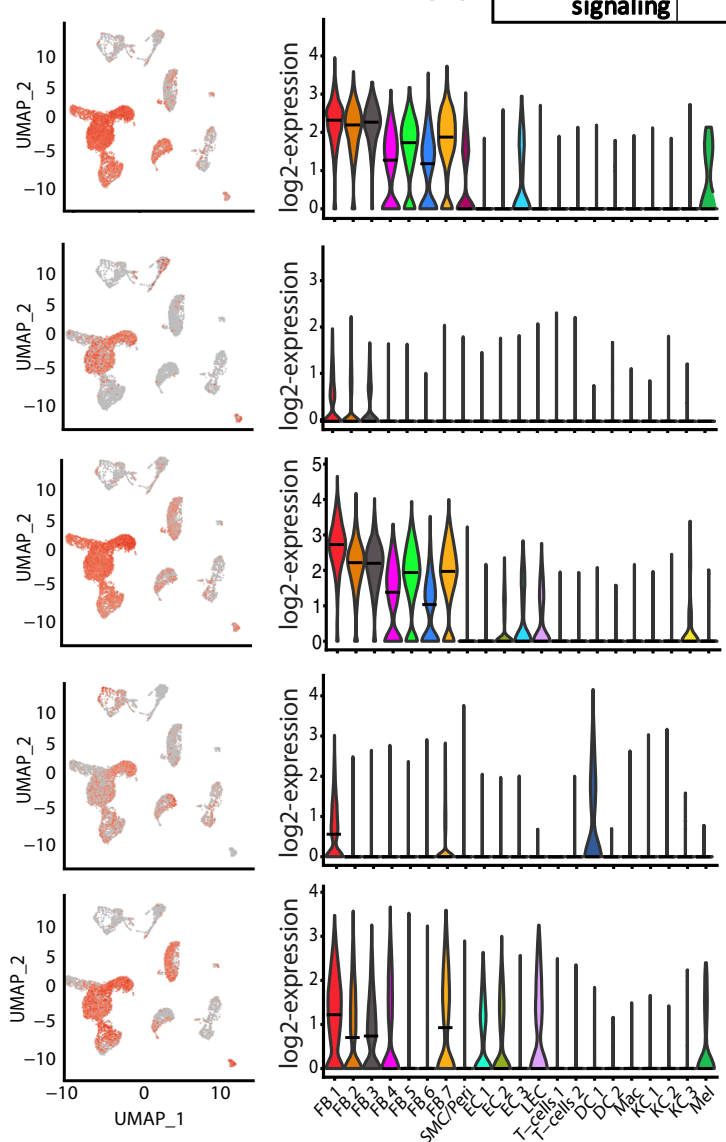

(J)

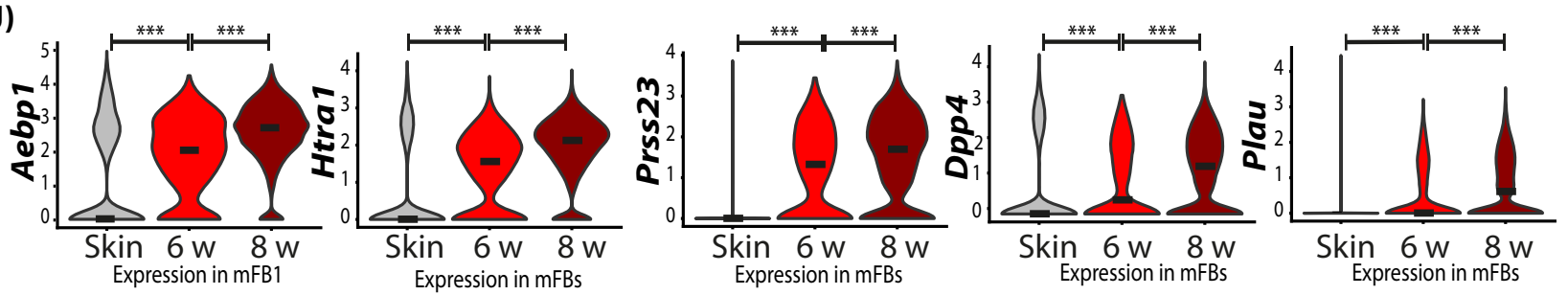

to identify comparable variations in T-cell subsets in human mature hypertrophic scars (Figure S13). It is therefore conceivable that these T-cell subsets play a role in initial scar formation processes rather than in established mature hypertrophic scars. In addition, species-dependent variances cannot be ruled out. Of note, Serpinb2, a specific urokinase inhibitor was downregulated in both species in specific T-cell subsets. Less endogenous urokinase inhibitors in scars might be an additional explanation for the high efficacy of BC-11, which was not only found in $\mathrm{T}$ cells but also in several other cell clusters in human scars and mouse scars (Figure S14). Together, these and many other novel factors identified in our study could be important, decisive molecules for the development and/or maturation of hypertrophic scars. Thus, our study has built a basis for future studies 
Fig. 5 Comparing human scar gene expression and mouse scar maturation identifies mutual drivers of skin fibrosis. A Illustration of computational basis for comparison human and mouse. Human cluster subFB1 vs remaining scar FBs significantly (adj. $p$-value $<0.05$ ) regulated genes were compared with mouse scar FBs 8 weeks vs 6 weeks significantly regulated genes. B Venn diagrams of human and mouse up- (upper panel) and down- (lower panel) regulated genes. C Table of mouse and human mutually up and $\mathbf{D}$ downregulated genes. E-I Violin plots and feature plots of serine proteases in mouse skin and scars. Vertical lines in violin plots represent maximum expression, shape of each violin represents all results, and width of each violin represents frequency of cells at the respective expression level. J Feature plots and violin plots of serine proteases in human skin and scar. AEBP1 (adipocyte enhancerbinding protein 1) $(p=2.22 \mathrm{e}-16, p=2.22 \mathrm{e}-16)$, DPP4 (dipeptidyl-peptidase 4$)(p=6.8 \mathrm{e}-9, p=1.1 \mathrm{e}-15)$, HTRA1 (high-temperature requirement A serine peptidase 1) $(p=2.22 \mathrm{e}-16, p=2.22 \mathrm{e}-16)$, PLAU (urokinase) $(p=2.22 \mathrm{e}-16, p=2.22 \mathrm{e}-16)$, PRSS23 (serine protease 23) $(p=2.22 \mathrm{e}-16, p=4 \mathrm{e}-14)$. In violin plots, dots represent individual cells, $y$-axis represents log2 fold change of the normalized genes and log-transformed single-cell expression. Vertical lines in violin plots represent maximum expression, shape of each violin represents all results, and width of each violin represents frequency of cells at the respective expression level. In feature plots, normalized log expression of the respective gene is mapped onto the UMAP-plot. Color intensity indicates level of gene expressions. UMAP, uniform manifold approximation and projection. A two-sided Wilcoxon-signed rank test was used in R. NS $p>0.05$, ${ }^{\star} p<0.05,{ }^{\star \star} p<0.01,{ }^{\star \star \star} p<0.001$.

describing the role of these molecules in skin scarring and tissue fibrosis.

Our combined study of human mature hypertrophic scars and scar maturation in mice identified a group of serine proteases as key player for scar development and maturation. Although DPP4positive FBs have previously been identified as the main source of ECM production in the skin ${ }^{50}$, and urokinase has been shown to be involved in lung fibrosis ${ }^{19}$, their roles in myofibroblast differentiation and production of ECM are still unclear. Our finding that siRNA-mediated gene knockdown and addition of specific DPP4 and urokinase inhibitors to TGF $\beta 1$-stimulated FBs almost completely inhibited myofibroblast differentiation and upregulation of matrix proteins was striking. Sitagliptin, the here used DPP4 inhibitor, is an effective drug widely used for the treatment of diabetes mellitus ${ }^{51}$. Recently, Li et al. showed that exposure of FBs derived from hypertrophic scars to high glucose led to activation of the IGF/Akt/mTOR signaling pathway, suggesting a possible mechanism by which gliptins interfere with fibrotic processes $^{52}$. Based on our study, it might be very interesting to systemically evaluate differences in scar formation and scar quality of diabetic patients treated with either gliptins or other drugs with serine protease inhibitory action. Indeed, an initial investigation on hypertrophic scar formation in Japanese patients receiving gliptins showed already promising results ${ }^{53}$. As gliptins are already approved for clinical use, an off-label topical application including non-diabetic patients would be a promising step forward to fully elucidate its efficacy on skin scarring.

The urokinase inhibitor BC-11 showed more pronounced effect on scar formation compared to sitagliptin. Strikingly, BC11 also inhibited the expression of both, PLAU and DPP4. The exact underlying mechanism needs further investigations; however, the combined action of $\mathrm{BC}-11$ on both serine proteases might explain its better performance on collagen deposition in vivo. So far BC-11 has only been used in vitro, and further in vivo testing for efficacy and safety is still required. Inhibition of urokinase to attenuate tissue fibrosis per se might appear counterintuitive, as urokinase facilitates fibrinolysis and regulates ECM-turnover, eliciting anti-fibrotic action ${ }^{54}$. However, literature on urokinase inhibitors and fibrosis is contradictory. The best investigated endogenous urokinase-inhibitor, plasminogen activator inhibitor-1 (PAI-1, SERPINE1), was found to cause excessive matrix deposition after injury ${ }^{55}$. By contrast, and in line with our results, inhibition of urokinase by PAI-1 suppressed profibrotic response in FBs from fibrotic lungs and prevented cardiac fibrosis in mice ${ }^{18}$. Therefore, our study suggests the use of urokinase inhibitor BC-11 as a possible therapeutic strategy for the treatment of skin scars. Further studies are necessary to fully elucidate its efficacy in vivo.

Surprisingly, our analyses revealed no influence of the inhibitors and knockdown of the serine proteases on the canonical
TGF $\beta 1$ signaling pathway. Although DPP4 inhibition has previously been demonstrated to directly inhibit canonical TGF $\beta$ signaling via Smad2 in renal fibrosis ${ }^{56}$ and TGF $\beta$-mediated myoFB-differentiation by interfering with ERK signaling 57 , we were not able to confirm these mechanisms in skin FBs. Regarding BC-11, we found a significant activation of GSK $3 \alpha / \beta$ in TGF $\beta 1$-stimulated FBs. Since GSK3 $\beta$ was previously found to interact with $\mathrm{WNT} / \beta$-catenin signaling 58,59 , and deletion of GSK3 $\beta$ induced a profibrotic myofibroblast phenotype in isolated cardiac FBs in mice ${ }^{43}$, the activation of GSK $3 \alpha / \beta$ suggests a counter-regulation of TGF $\beta 1$ signaling. It is therefore conceivable that $\mathrm{BC}-11$, at least partially, exerts its anti-fibrotic action via activation of GSK3 $\alpha / \beta$. Deciphering the exact underlying mechanism by which the inhibitors interfere with TGF $\beta$ signaling will be the scope of further studies.

In this study, we analyzed human hypertrophic scars and mouse scar formation on a single-cell level. However, several limitations should be considered. Due to the high costs and the fact that scRNAseq yields large datasets of tens of thousands of cells, thereby smoothening donor and technical variances ${ }^{60}$, low donor numbers are usually justifiable ${ }^{61-63}$. Nevertheless, the relatively small sample size in our study should be considered as a limitation of our study. Differences in body sites between scar tissue and healthy skin, and the fact that healthy skin and scars were not taken from the same donors could affect comparability of the data. However, a recent study by Ascension et al. ${ }^{64}$, comparing different single-cell datasets of skin samples from different body regions showed that the major FB populations were consistently present in all donors and body sites, suggesting high comparability.

Furthermore, there are certainly considerable differences between human and murine wound healing; while mouse wounds heal predominantly via contraction promoted by the subcutaneous panniculus carnosus, de novo formation and deposition of ECM and subsequent re-epithelialization prevails in human wound healing ${ }^{65}$. However, a study assessing contribution of epithelialization and contraction in mice found that each accounted for $40-60 \%$, and that mouse wound models can thus be considered a valid model also for human wound healing ${ }^{66}$. Moreover, our mouse scarring model does not fully reflect the pathological fibrotic state of human hypertrophic scars. Although mouse models for hypertrophic scars, e.g., subcutaneous bleomycin injection ${ }^{67}$, or tight-skin mice ${ }^{68}$ have been described, their transcriptome comparability with human hypertrophic scars is not well investigated. We therefore suggest that future studies testing the efficacy of serine protease inhibitors should be performed in large animal models, e.g., pigs, which better reflect the pathology of human hypertrophic scars ${ }^{68}$. In our experimental model, creams containing protease inhibitors were topically applied on wounds and scar tissue after complete wound closure. 

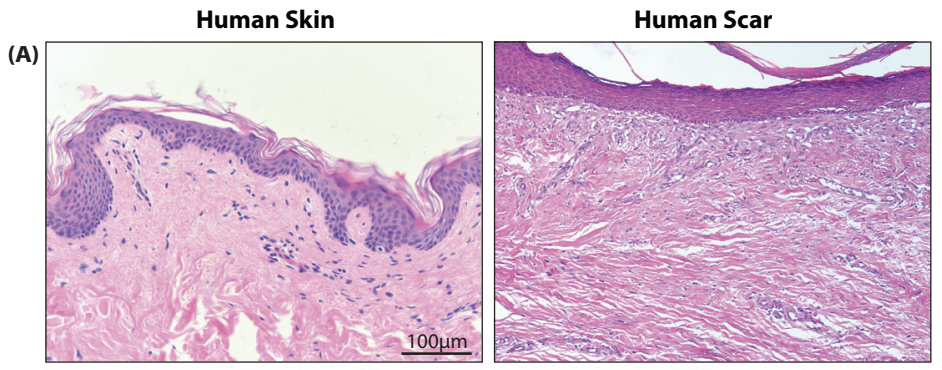

(B)
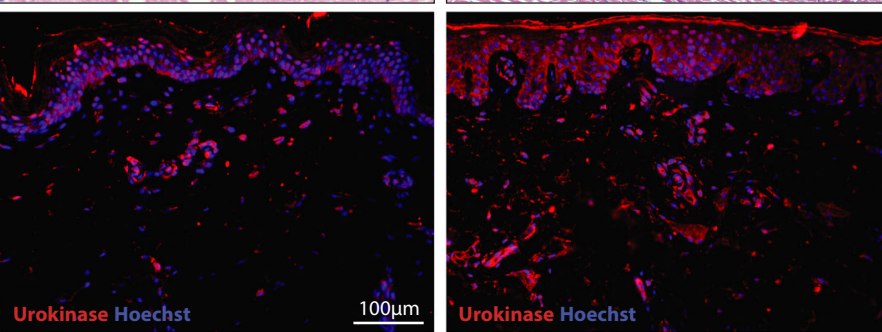

(C)
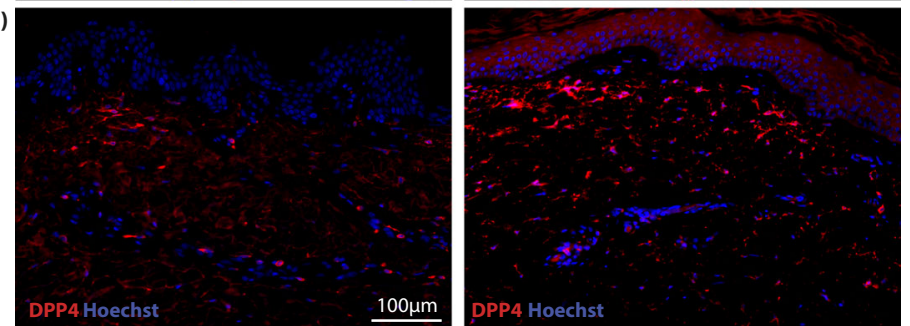

(D)

Urokinase human dermis

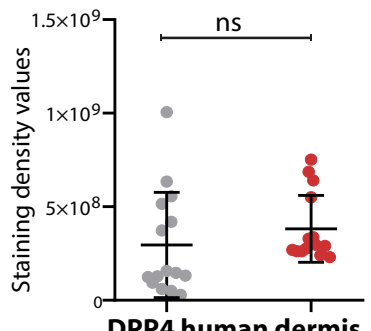

Urokinase human epidermis

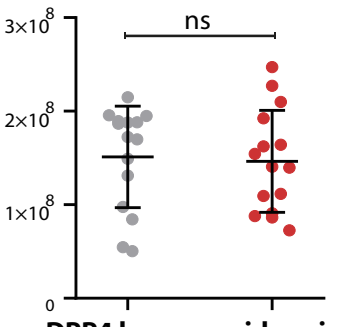

(E)

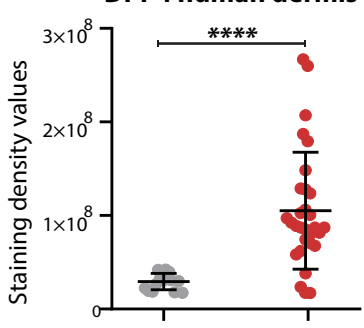

DPP4 human epidermis

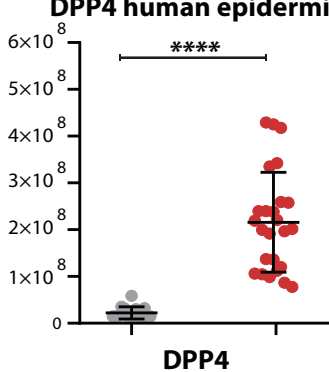

(F)

Urokinase

(G)

human skin lysate
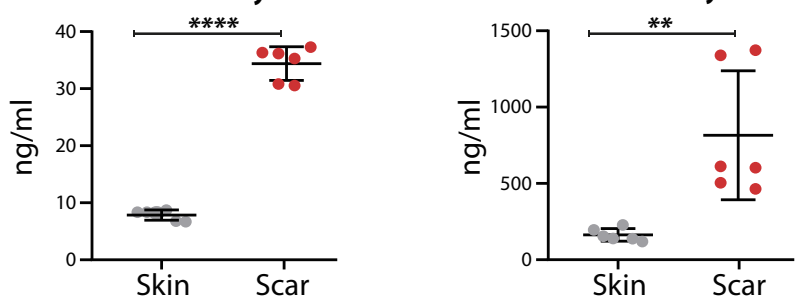

Fig. 6 Immunofluorescence staining confirms elevated expression of PLAU and DPP4 in human and mouse skin and scar. A H\&E staining of human skin and scar; immunofluorescent staining of B Urokinase and C DPP4 in human skin and scar. Quantification of staining intensity separate for epidermis and dermis for $\mathbf{D}$ urokinase ( $p=0.329$ dermis, $p=0.815$ epidermis) and E DPP4 ( $p<0.0001$ dermis, $p<0.0001$ epidermis). For all stainings, $n=2-3$ normal skin samples were analyzed, and $n=4$ scars. From each sample, five regions of interest per sample were quantified. ELISA from human whole skin ( $n=6$ ) and scar $(n=6)$ lysate for $\mathbf{F}$ urokinase $(p<0.0001)$ and $\mathbf{G}$ DPP4 ( $p=0.0037)$ is shown. Statistical significance was tested using two-tailed unpaired Student $t$-test. Lines and error bars indicate mean and standard deviation. NS $p>0.05,{ }^{\star} p<0.05,{ }^{\star \star} p<0.01,{ }^{\star \star \star} p<0.001$. Source data are provided as a Source data file. 
(A)

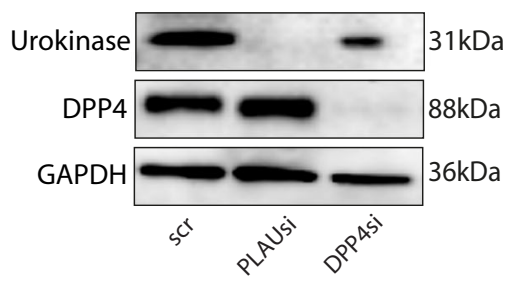

(B)

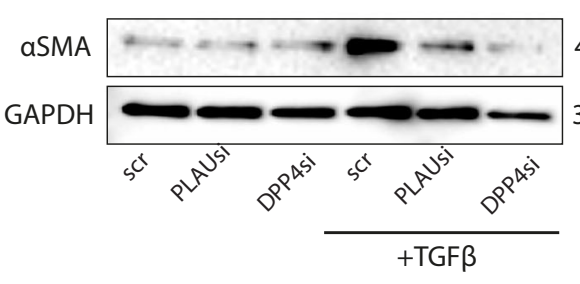

(D)
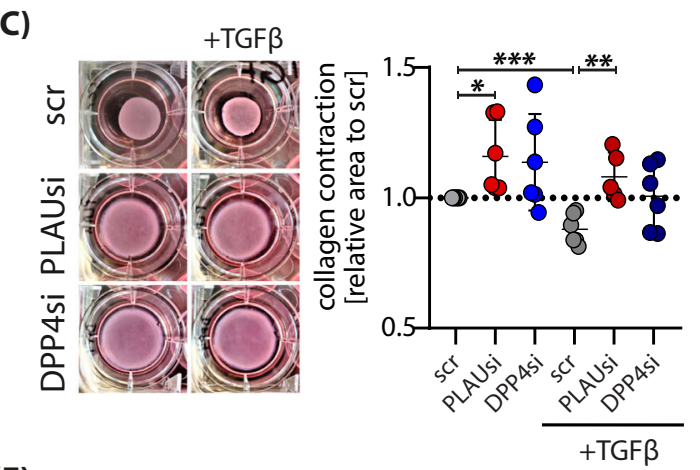

COL1a1
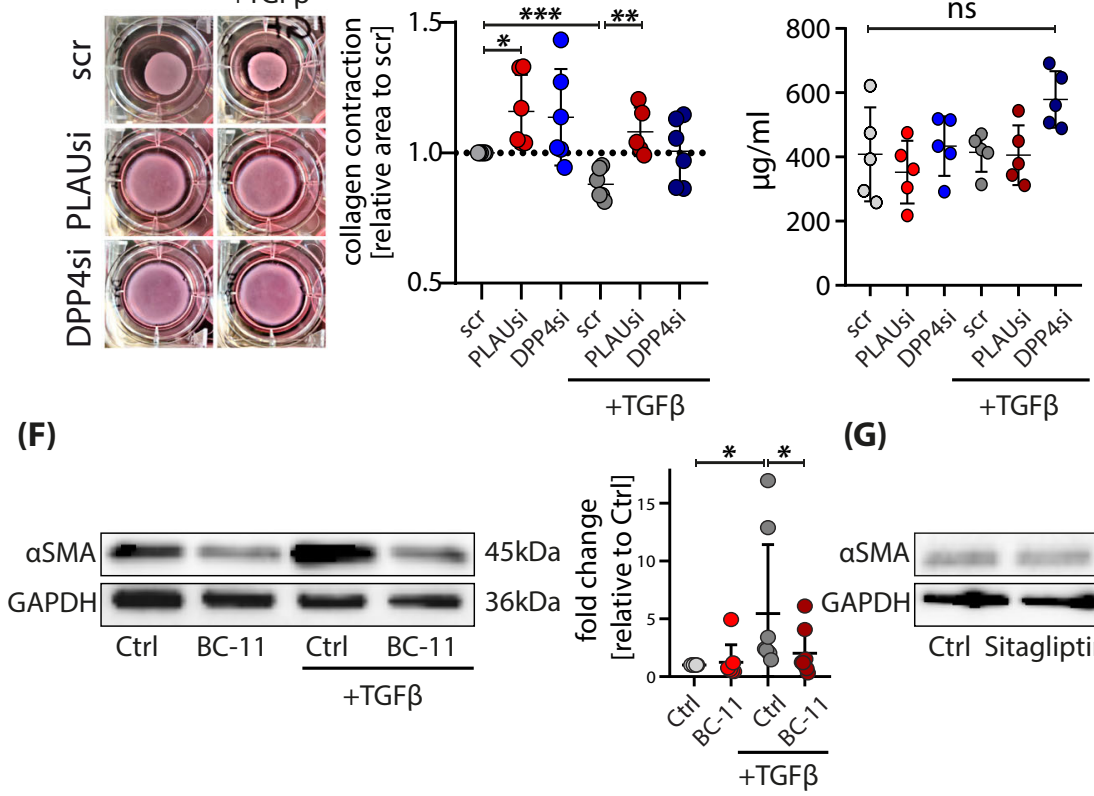

(G)
$45 \mathrm{kDa}$ $36 \mathrm{kDa}$

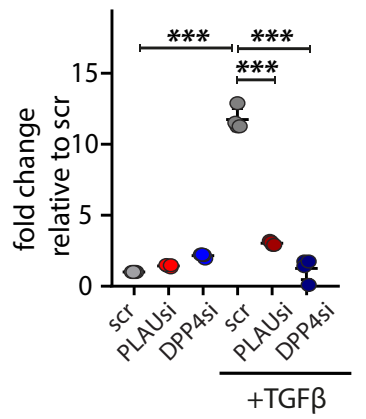

(H)

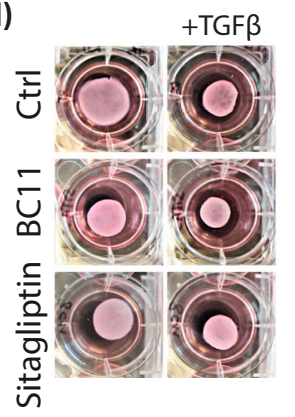

(I)

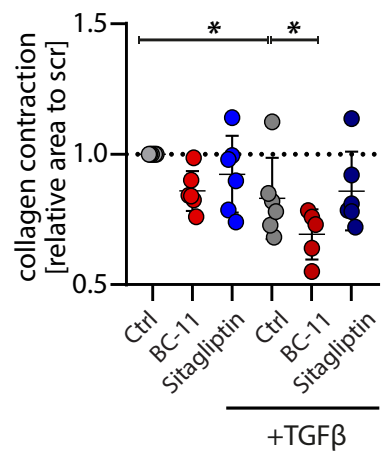

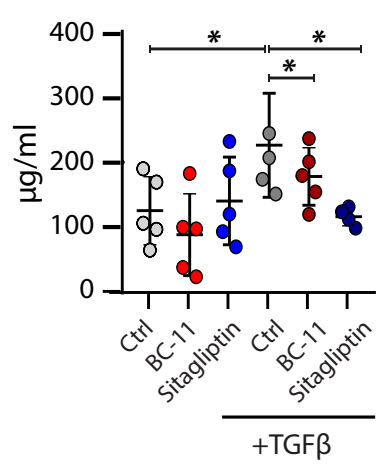

(E)

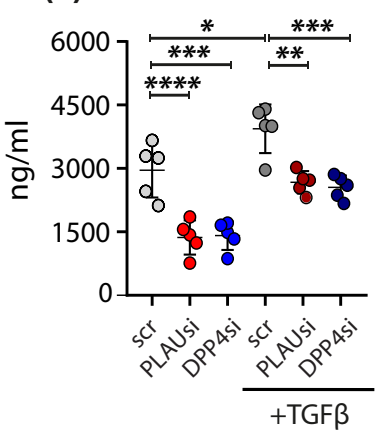


Fig. 7 Knockdown and pharmacological inhibition of DPP4 or urokinase prevents TGF $\beta$-induced myofibroblast differentiation. A, B Western blot of primary FBs after knockdown of PLAU or DPP4. B Western blot of primary FBs after knockdown of DPP4 or PLAU stimulated with active TGF $\beta 1$ for $24 \mathrm{~h}$ to differentiate FBs into alpha-smooth muscle actin-expressing ( $\alpha \mathrm{SMA}$ ) myofibroblasts, and western blot quantification $(n=3)$. $\mathrm{scr} v \mathrm{vs} \mathrm{scr}+\mathrm{TGF} \beta 1, p=0.0006$; scr+TGF $\beta 1$ vs PLAUsi+TGF $\beta 1 p=0.0010$; scr+TGF $\beta 1$ vs DPP4si + TGF $\beta 1 p=0.0017$. C Collagen contractility with FBs after knockdown of PLAU or DPP4 and stimulation with or without active TGF $\beta 1$. scr vs PLAUsi $p=0.0194$; scr vs scr+TGF $\beta 1 p=0.0005$; scr + TGF $\beta 1$ vs PLAUsi+TGF $\beta 1=0.0018$. D Collagen I $(p>0.05)$ and $\mathbf{E}$ fibronectin (scr vs scr+TGF $\beta 1, p=0.0193$; scr vs PLAUsi $p<0.0001$; scr vs DPP4si $p=0.0001$; scr + TGF $\beta 1$ vs PLAUsi + TGF $\beta 1=0.0017$; scr+TGF $\beta 1$ vs DPP4si + TGF $\beta 1=0.0006$ ) concentrations in supernatants of TGF $\beta 1$-stimulated primary skin FBs after knockdown with PLAU or DPP4. F, G Western blot of primary FBs stimulated with active TGF $\beta 1$ for $24 \mathrm{~h}$ to differentiate FBs into alpha-smooth muscle actin-expressing ( $\alpha \mathrm{SMA}$ ) myofibroblasts, and quantification of western blot $(n=5-6)$. Myofibroblast differentiation inhibited with $\mathbf{F}$ urokinase inhibitor BC-11 (Ctrl vs Ctrl+TGF $\beta 1 p=0.049$, Ctrl+TGF $\beta 1$ vs BC$11+$ TGF $\beta 1 p=0.020$ ) or G DPP4 inhibitor Sitagliptin (Ctrl vs Ctrl+TGF $\beta 1 p=0.0183$, Ctrl+TGF $\beta 1$ vs Sitagliptin+TGF $\beta 1 p=0.0356$ ). H Collagen contractility with FBs after inhibition with BC-11 or Sitagliptin and stimulation with or without active TGF $\beta 1$ (Ctrl vs Ctrl+TGF $\beta 1 p=0.024 ; \mathrm{Ctrl}+\mathrm{TGF} \beta 1$ vs BC-11 + TGF $\beta 1 p=0.037$ ). I Collagen I (Ctrl vs Ctrl+TGF $\beta 1 p=0.0465$; Ctrl+TGF $\beta 1$ vs BC-11 + TGF $\beta 1 p=0.021$ ) or J fibronectin (Ctrl vs Ctrl+TGF $\beta 1 p=0.0009 ;$ Ctrl vs Sitagliptin +TGF $\beta 1$ $p=0.0002$ ) in supernatants of stimulated primary skin FBs, detected by enzyme-linked immunosorbent assay (ELISA). Quantification from western blot was calculated by pixel density measurement in ImageLab, adjusted to GAPDH and normalized to respective Ctrl values. Experiments were performed in duplicates of $n=5$ each. Whiskers represent range maximum and minimum values with $<1.5$ interquartile range, boxes represent 25 th- 75 th quartiles, line represents mean. Statistical significance was tested using two-way ANOVA with Tukey post-test. NS $p>0.05,{ }^{\star} p<0.05,{ }^{\star \star} p<0.01,{ }^{\star \star *} p<0.001$. Source data are provided as a Source data file.

Table S1). Scars were classified as hypertrophic, pathological scars according to POSAS $^{70}$ by a plastic surgeon. Only mature scars, which had not been treated before and persisted for more than 2 years were used for all experiments. All donors had no known chronic diseases and received no chronic medication. The quality of scar tissue was assessed by histological analysis. No adjacent normal skin was observed in any of the scar samples. Healthy skin $(n=3)$ was obtained from female donors between 25 and 45 years from surplus abdominal skin removed during elective abdominoplasty.

Mouse full skin wounding and scar maturation. Female Balb/c mice bred at the animal facility of the Medical University of Vienna (Himberg, Austria) were housed under specific-pathogen-free conditions at $22 \pm 2^{\circ} \mathrm{C}$ room temperature and $55 \pm 10 \%$ humidity, with $12 \mathrm{~h} / 12 \mathrm{~h}$ light/dark cycles and food and water access ad libidum. Female mice were used due to easier handling and better experimental compliance, which was necessary to enable frequent handling and application of treatment. For full-thickness skin wounds, mice were anesthetized with $100 \mathrm{mg} / \mathrm{kg}$ Xylazin and $5 \mathrm{mg} / \mathrm{kg}$ ketamin (both Sigma-Aldrich, St. Louis, MO, USA) intraperitoneally. Postoperative analgesia was provided with $0.1 \mathrm{mg} / \mathrm{kg}$ Buprenorphin (Temgesic ${ }^{\oplus}$, Indivior Inc., North Chesterfield, VA, USA) subcutaneously and $0.125 \mathrm{mg} / \mathrm{ml}$ Piritramid (Janssen-Cilag Pharma, Vienna, Austria) in drinking water ad libidum. A $9 \times 9 \mathrm{~mm}^{2}$ area was marked on shaved backs and excised with sharp scissors. The wounds were left to heal uncovered without any further intervention. Mice were sacrificed 6 or 8 weeks after wounding, and scar tissues were isolated. Four-millimeter biopsies were taken from the scar tissue and analyzed individually for scRNAseq as described below. The quality of scar tissue was assessed by histological analysis. Samples with a maximum of $20 \%$ normal adjacent skin were used for further analyses.

Serine protease inhibitor treatment. Mouse full-thickness skin wounds were induced as described above. Ultrasicc/Ultrabas ointment (1:2; Hecht-Pharma, Bremervörde, Germany) was used as carrier substance for all treatments. Four parts Ultrasicc/Ultrabas and 1 part water were mixed and used as control treatment. For protein inhibitors, Sitagliptin (final concentration $1 \mathrm{mM}$ ) or BC-11 (final concentration $5 \mathrm{mM}$ ) were dissolved in water and mixed with the ointment. Immediately after wounding, mice were treated with control or inhibitors by applying $100 \mu \mathrm{l}$ ointment on each wound. After application, mice were put individually in empty cages without litter for $30 \mathrm{~min}$ and monitored closely to prevent immediate removal of the treatments and allow sufficient tissue resorption. Scabs were left intact to prevent wound infections. Mice were treated daily for the first $7 \mathrm{~d}$, and thrice a week for 7 weeks. After scar formation, $4 \mathrm{~mm}$ biopsies of the scar tissue were taken and cut in half. One half each scar sample was used for histological analysis, and the other biopsy halves from each treatment group were pooled and analyzed together with scRNAseq as described below.

Single-cell isolation and fluorescence-activated cell sorting (FACS). Biopsies from human skin, human scars, and from naturally matured or treated mouse scar tissue were enzymatically digested with MACS Miltenyi Whole Skin Dissociation Kit (Miltenyi Biotec, Bergisch-Gladbach, Germany) for $2.5 \mathrm{~h}$ according to the manufacturer's protocol. After processing on a GentleMACS OctoDissociator (Miltenyi), cell suspensions were passed through a $70 \mu \mathrm{m}$ and a $40 \mu \mathrm{m}$ filter and stained with DAPI nuclear dye. Cells were sorted on a MoFlo Astrios high-speed cell sorting device (Beckman-Coulter, Brea, CA, USA), and only DAPI-negative cells, representing viable cells, were used for single-cell RNAseq (Figure S1).
Generation of single-cell gel-bead in emulsions (GEMs) and library preparation. Immediately after sorting, viable cells were loaded on a 10X-chromium instrument (single-cell gene expression 3'v2/3, 10X Genomics, Pleasanton, CA, USA) to generate GEMs. GEM-generation, library preparation, RNA-sequencing, demultiplexing, and counting were done by the Biomedical Sequencing Core Facility of the Center for Molecular Medicine (CeMM, Vienna, Austria). Sequencing was performed on an Illumina HiSeq 3000/4000 (Illumina, San Diego, CA, USA) with 3 samples per lane, $2 \times 75 \mathrm{bp}$, and paired-end sequencing.

Cell-gene matrix preparation and downstream analysis. Raw sequencing files were demultiplexed, aligned to the human or mouse reference genome (GrCh38/ $\mathrm{mm} 10$ ) and counted using the Cellranger pipelines (Cellranger v3, 10X Genomics). The resulting cell-gene matrices were processed using the 'Seurat'-package (Seurat v3.1.0, Satija Lab, New York, NY, USA) in R-studio in R (R v3.6.2, The R Foundation, Vienna, Austria). From each sample, unwanted variations and low-quality cells were filtered by removing cells with high and low $(>3000$ and $<200)$ unique molecular identifier (UMI)-counts. First, healthy skin and scar samples were integrated separately to avoid clustering according to donors, and for batch correction. Subsequently, skin and scar data were integrated again into one dataset. Data integration was performed according to the recommended workflow by Butler et al. and Stuart et al. ${ }^{60,71}$. After quality control comparing all donors, we obtained transcriptome data from a total of 25,083 human skin and scar cells, with a median of 24,943 reads and 851 detected genes per cell. In mice, we obtained data from 6561 cells 6 weeks after wounding, and 9393 cells 8 weeks after wounding. The samples displayed a median of 24,774 reads per cell, and median of 1969 detected genes per cell. After quality control, all mouse samples were integrated together in one integration step. In both datasets, normalized count numbers were used for differential gene expression analysis, for visualization in violin plots, feature plots, dotplots, and heatmaps, when displaying features that vary across conditions, as recommended by current guidelines ${ }^{72}$. In both datasets, cell types were identified by well-established marker gene expression (Figures S2A and S4A). For identification of differentially expressed genes (DEGs), normalized count numbers were used, including genes present in the integrated dataset to avoid calculation of batch effects. As keratin and collagen genes were previously found to contaminate skin biopsy datasets and potentially provide a false-positive signal ${ }^{73}$, these genes (COL1A1, COL1A2, COL3A1 and KRT1 KRT5, KRT10, KRT14, KRTDAP) were excluded from DEG calculation in non-fibroblast clusters (collagens) or nonkeratinocyte clusters (keratins), respectively. Moreover, genes Gm42418, Gm17056, and Gm26917 caused technical background noise and batch effect in mouse scRNAseq, as described before ${ }^{74}$, and were thus excluded from the dataset.

Pseudotime analyses. Pseudotime analyses, trajectory construction, and calculation of pseudotime-dependent gene expression were performed in Monocle2 (Monocle2, v2.14.0, Trapnell Lab, University of Washington, Seatlle, WA, USA $)^{35,75}$. From the integrated FB subset Seurat-object, data were converted into a monocle-compatible CellDataSet. Analysis was then performed according to the recommended pipeline. Cells with mRNA counts two standard deviations above or below the mean were excluded. Size factors and dispersions were estimated, tSNEreduction and clustering were performed ${ }^{35,36,75}$. As input for pseudotime ordering, differentially expressed genes between skin and scar were used, and trajectories were constructed with DDRTree (R-package 'DDRTree' v0.1.5, 2015) ${ }^{36}$.

Gene ontology (GO)-networks. Gene lists of significantly regulated genes (adjusted $p$-value $<0.05$, average log fold change [avg_logFC] $>0.1$ ) were imputed in ClueGO v2.5.576 plug-in in Cytoscape v3.7. $2^{77}$ with medium GO-specificity, with 
(A)
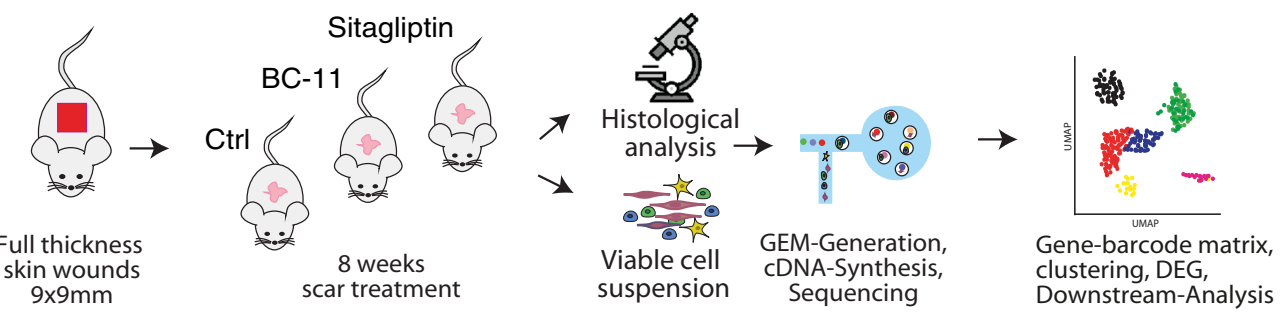

(B)

Ctrl BC-11 Sitagliptin

(C)

Wound area
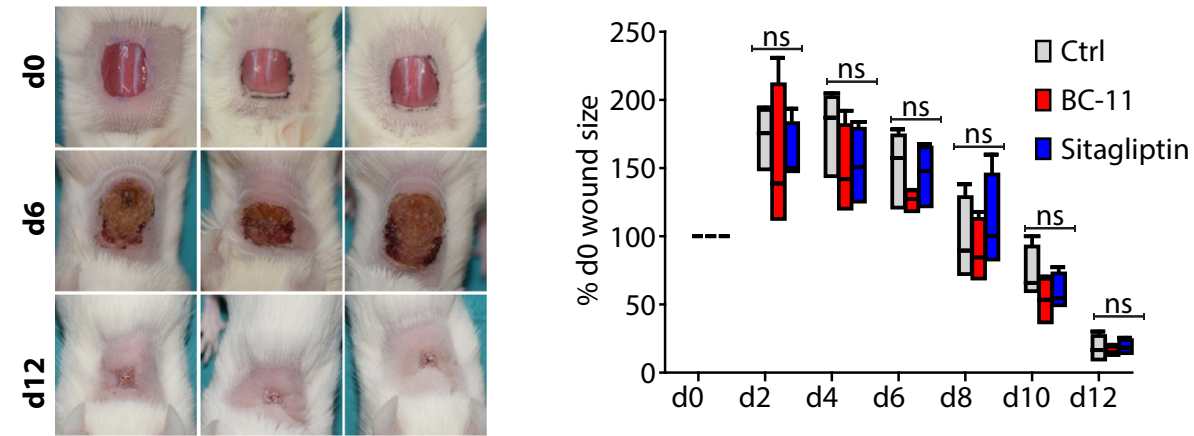

(D)

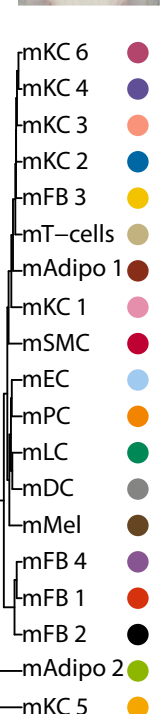

(E)

Ctrl

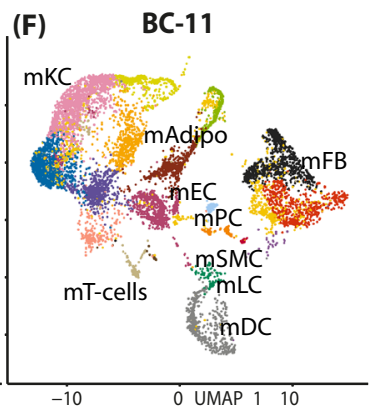

(G) Sitagliptin
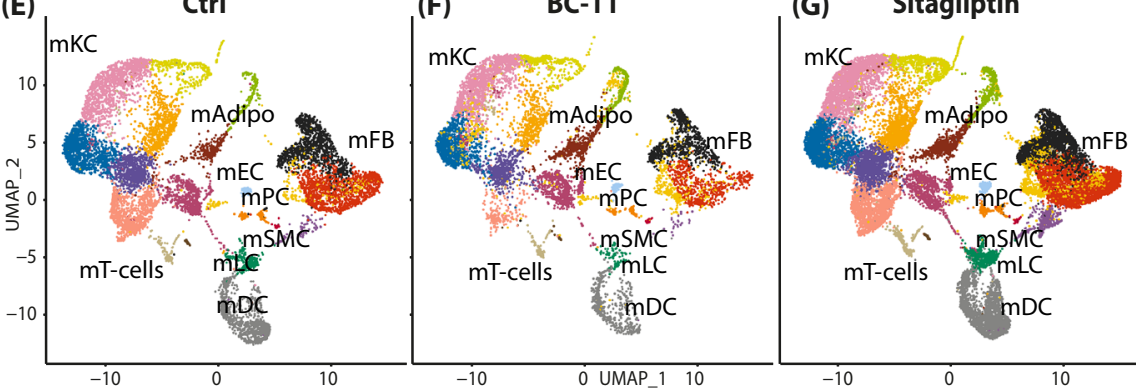

(H)
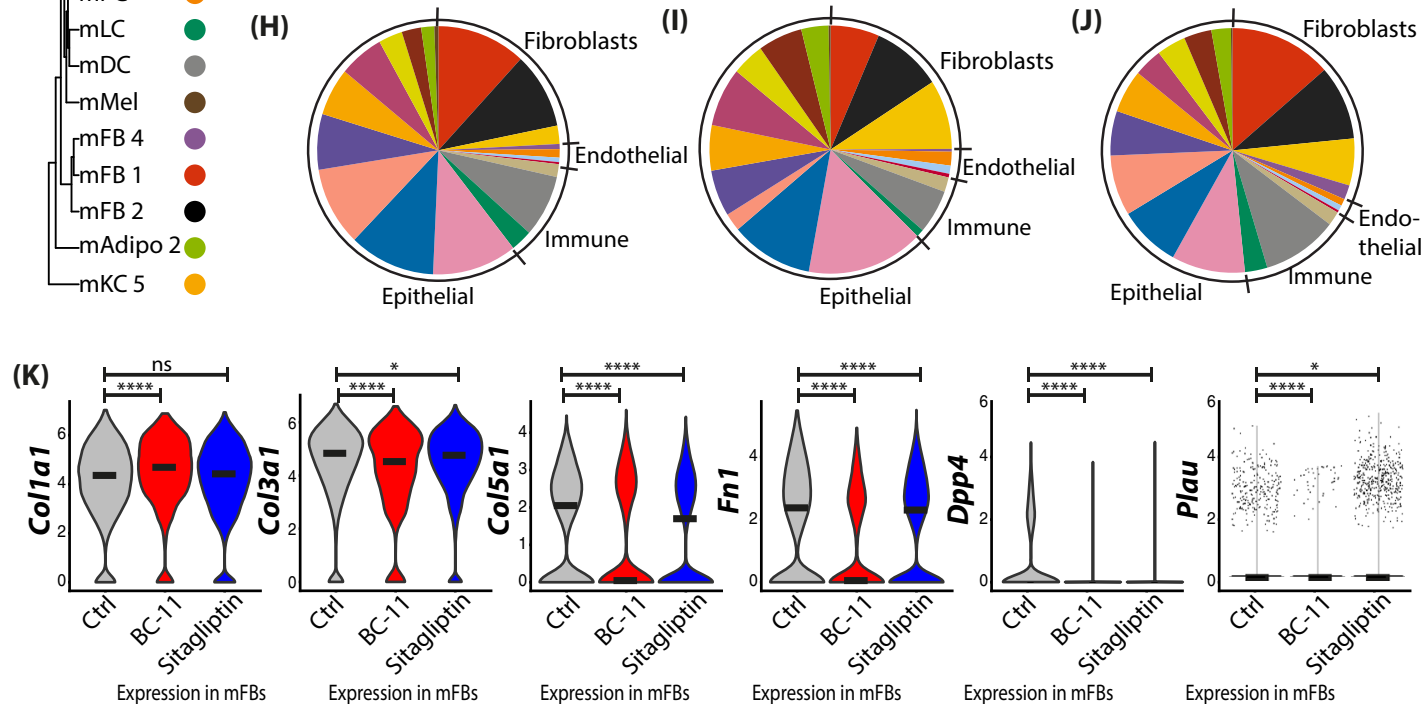

Expression in $\mathrm{mFB}$

Expression in $\mathrm{mFB}$

Expression in $\mathrm{mFBs}$

GO-term fusion, and only significant $(P$ value $<0.05)$ GO terms are depicted as circles, whereby circle size correlates with $P$ value, and lines represent functional connection of respective GO terms.

Immunofluorescence staining. Immunofluorescence staining on formalin-fixed, paraffin-embedded (FFPE) sections of skin and scar tissue were performed according to the protocol provided by the respective antibody manufacturer as described previously ${ }^{78}$. In brief, sections were deparaffinized in xylene and alcohol, antigen retrieval was performed with $\mathrm{pH} 6$ citric acid buffer, sections were washed in phosphate-buffered saline, and incubated with antibodies overnight at $4{ }^{\circ} \mathrm{C}$. Sections were washed again, and incubated with secondary antibodies, blocking sera of secondary antibodies species, and Hoechst, for $1 \mathrm{~h}$ at room temperature. Antibodies were used as indicated in Table S2. After the last wash, sections were mounted in aqueous mounting medium. Stainings were photographed using an Olympus BX63 microscope (Olympus, Tokyo, Japan) with Olympus CellSens Dimension v2.3 (Olympus) software with standardized exposure time for all samples. Staining intensity was quantified separately in dermis and epidermis using ImageJ v1.53c $\mathrm{c}^{79}$. For dermal quantification, regions of interest (ROIs) of $70 \times 70$ pixels were directly adjacent and parallel to the epidermis, contained no hair follicles or blood vessels, and were obtained from all regions of the specimen. For epidermal quantification, ROIs of $30 \times 30$ pixels located within the epidermis from all regions of each specimen. The total corrected fluorescence was measured by subtracting background values from area integrated density in the respective ROI. 
Fig. 8 In vivo application of BC-11 or Sitagliptin reduces expression of ECM and serine proteases. A Workflow of mouse scarring and serine protease inhibitors. Biopsies of $n=4$ mice per group were pooled for scRNAseq. B Images of wound healing in control or inhibitor-treated mice after 9,6 , and 12 days. C Quantification of wound area every second day after wounding. Four mice per group with three measurements per timepoint per mouse were analyzed. Wound area of $\mathrm{dO}$ of every mouse was used as reference, and area was compared as percent of original wound size. Boxes indicate first and third quartile, whiskers indicate min and max, line indicates median. Statistical significance was tested using two-way ANOVA with Tukey post-test. D Phylogenetic clustertree calculated based on unsupervised UMAP-clustering. E-G UMAP-plots of mouse scar tissue, split by timepoint, after integration of all samples, identifying four fibroblast clusters (mFB1-4), smooth muscle cells and pericytes (mPC/SMC), endothelial cells and lymphatic endothelial cells ( $\mathrm{mEC} / \mathrm{LEC}$ ), T cells, dendritic cells ( $\mathrm{mDC}$ ), Langerhans cells ( $\mathrm{mLC}$ ), nine keratinocyte clusters (KC1-9), adipocytes (mAdipo), and melanocytes (Mel). H-J Pie charts show relative numbers of cells in clusters, split by treatment. K Violin plots of ECM-associated genes. Col1a1 Ctrl vs BC-11 $p=4.8 \mathrm{e}-16$, Ctrl vs Sitagliptin $p=0.3$; Col3a1 Ctrl vs BC-11 $p=3.2 \mathrm{e}-12$, Ctrl vs Sitagliptin $p=0.028$; Col5a1 Ctrl vs BC-11 $p=1 \mathrm{e}-9$, Ctrl vs Sitagliptin $p=1.4 \mathrm{e}-9 ;$ Fn1 Ctrl vs $\mathrm{BC}-11 p=2.22 \mathrm{e}-16$, Ctrl vs Sitagliptin $p=8.6 \mathrm{e}-5 ;$ Dpp 4 trl vs BC-11 $p=2.22 \mathrm{e}-16$, Ctrl vs Sitagliptin $p=8.3 \mathrm{e}-11 ;$ Plau Ctrl vs BC-11 $p=5.04 \mathrm{e}-6, \mathrm{Ctrl}$ vs Sitagliptin $p=0.022$; vertical lines in violin plots represent maximum expression, shape of each violin represents all results, and width of each violin represents frequency of cells at the respective expression level. A two-sided Wilcoxon-signed rank test was used in R. UMAP, uniform manifold approximation and projection. NS $p>0.05,{ }^{\star} p<0.05,{ }^{\star \star} p<0.01,{ }^{\star \star \star} p<0.001$. using one-way ANOVA with Tukey post-test. NS $p>0.05,{ }^{\star} p<0.05$, ${ }^{\star \star} p<0.01,{ }^{\star \star *} p<0.001$. Source data are provided as a Source data file.

Picrosirius red staining. Picrosirius Red staining was performed according to the manufacturer's protocol of the staining kit (ab150681, Abcam, Cambridge, UK).

Isolation of primary skin fibroblasts. Five mm biopsies were taken from fresh abdominal skin, washed in phosphate-buffered saline (PBS), and incubated in $2.4 \mathrm{U} / \mathrm{ml}$ Dispase II (Roche, Basel, Switzerland) overnight at $4{ }^{\circ} \mathrm{C}$. The next day, epidermis was separated from dermis, and dermis was incubated with Liberase TM (Merck Millipore, Burlington, MA, USA) in Dulbeccos modified eagle medium (DMEM, Thermo Fisher Scientific, Waltham, MA, USA) without supplements at $37^{\circ} \mathrm{C}$ for $2 \mathrm{~h}$. Next, the dermis was passed through $100 \mu \mathrm{m}$ and $40 \mu \mathrm{m}$ filters, rinsed with PBS, and cells were plated in a T175 cell culture flask. Medium was changed the next day, and then every other day until FBs reached $90 \%$ confluency. First passage FBs were used for TGF $\beta 1$-stimulation experiments.

TGFß1-induced myofibroblast differentiation. After the first passage, isolated primary FBs were plated in 6-well plates, supplied with DMEM $+10 \%$ fetal bovine serum (FBS, Thermo Fisher Scientific) and 1\% penicillin/streptomycin (Thermo Fisher Scientific) and grown until 100\% confluency. FBs were then stimulated with 10 ng/ml TGF $\beta 1$ (HEK-293-derived, Peprotech, Rocky Hill, NJ, USA), and with or without DPP4 inhibitor Sitagliptin $(10 \mu \mathrm{M})$ (Thermo Fisher Scientific) or urokinase-inhibitor BC-11 hydrobromide $(10 \mu \mathrm{M})$ (Tocris by Bio-Techne, Bristol, UK) for $24 \mathrm{~h}$. Supernatants were removed and medium and inhibitors were resupplied for another $24 \mathrm{~h}$. Supernatants were collected and stored at $-80{ }^{\circ} \mathrm{C}$ and cells were lysed in 1x Laemmli Buffer (Bio-Rad Laboratories, Inc., Hercules, CA, USA) for further analysis. To analyze signaling pathways, FBs were stimulated with TGF $\beta 1$ and inhibitors for $1 \mathrm{~h}$, and then harvested in 1x Laemmli Buffer with protease inhibitor (cOmplete, MiniProtease Inhibitor Cocktail Tablets, Roche, Basel, Switzerland) and phosphatase inhibitor (Pierce ${ }^{\mathrm{Tm}}$ Phosphatase Inhibitor Mini Tablets, Thermo Scientific).

siRNA-mediated gene silencing. Small interfering RNA (siRNA) transfection was conducted according to the previously published protocol ${ }^{80}$ with minor modifications. siRNAs targeting PLAU (\#HSS108076, Thermo Fisher Scientific) and DPP4 (\#HSS102892, Thermo Fisher Scientific) were used. Briefly, primary human FBs of 3 donors were transfected using Lipofectamine 2000 (Thermo Fisher Scientific). A total of $5 \mathrm{ml}$ of Opti-MEM medium (+L-Glutamine, 4-[2-hydroxyethyl]-1-piperazineethanesulfonic acid, Phenol Red; Gibco by Life Technologies) were mixed with $50 \mu \mathrm{l}$ of Lipofectamine 2000 and $65 \mu \mathrm{l}$ of a $20 \mu \mathrm{M}$ small interfering RNAs or scrambled control RNA (Low GC Duplex; Thermo Fisher Scientific). After $15 \mathrm{~min}$ incubation, the solution was added to $20 \mathrm{ml}$ DMEM medium and transferred to FBs. Protein and RNA samples were prepared $48 \mathrm{~h}$ after transfection.

Quantitative real-time PCR. Total RNA was prepared from fibroblast monolayers using TRIzol (Thermo Fisher Scientific) according to manufacturer's instructions. cDNA was synthesized using iScript ${ }^{\text {tw }} c$ DNA Synthesis Kit (Bio-Rad, Hercules, CA, USA) according to manufacturer's instructions. Relative quantification was performed by using the Light Cycler Master SYBR Green I kit (Roche Applied Science, Basel, Switzerlad) on a LightCycler480 II thermocycler (Roche). Primers were designed using the Primer3 software (version 0.4.0, https://bioinfo.ut.ee/primer30.4.0/) and synthesized by Microsynth AG (Balgach, Switzerland). Samples were normalized to $\beta$-2-microglobulin (B2M) levels as reference gene. Primers with the sequences indicated in Table S3 were used.

Gel contraction assay. Primary human FBs or FBs silenced for DPP4 or PLAU $\left(3 \times 10^{5}\right.$ fibroblasts per $\left.\mathrm{ml}\right)$ were mixed purified bovine collagen solution (PureCol, Advanced BioMatrix, San Diego, CA) and 10\% 10× Hanks' Balanced Salt Solution
(Thermo Fisher Scientific). Cell suspensions were poured into 6-well plates and allowed to solidify for $2 \mathrm{~h}$ at $37^{\circ} \mathrm{C}$ in a humidified atmosphere. After equilibration with DMEM medium overnight, the collagen gels containing knockdown fibroblasts were further incubated with DMEM and gels with normal fibroblast were either treated with sitagliptin $(10 \mu \mathrm{M})$ and BC-11 $(10 \mu \mathrm{M})$ or left untreated. Collagen gels were further maintained in the absence or presence of TGF $\beta 1(10 \mathrm{ng} / \mathrm{ml})$. After $48 \mathrm{~h}$, gels were photographed, and gel areas were calculated using ImageJ software.

Western blotting. Primary FBs were lysed in 1x Laemmli Buffer (Bio-Rad Laboratories, Inc.) and loaded on 4-15\% SDS-PAGE gels (Bio-Rad Laboratories, Inc.). Proteins were transferred on a nitrocellulose membrane (Bio-Rad Laboratories, Inc.), membranes were blocked in non-fat milk with $0.1 \%$ Tween 20 (SigmaAldrich for $1 \mathrm{~h}$, and incubated with antibodies as indicated in Table S2 at $4{ }^{\circ} \mathrm{C}$ overnight. After washing, membranes were incubated with horseradish-peroxidase conjugated secondary antibodies as indicated in Table S2 for $1 \mathrm{~h}$ at room temperature. Signals were developed with SuperSignal West Dura substrate (Thermo Fisher Scientific) and imaged with a Gel Doc XR + device (Bio-Rad Laboratories, Inc.). Quantification analysis was performed with the Volume tool in ImageLab 6.0.1 (Bio-Rad), adjusted to GAPDH expression, and normalized to respective Ctrl samples to calculate fold change to Ctrl.

Proteome profiling of signaling pathways. To analyze signaling pathways, we used a proteome profiler for human phospho-kinases (ARY003C, R\&D Systems, Biotechne, Minneapolis, MN, USA) according to the manufacturer's instructions.

Enzyme-linked immunosorbent assay (ELISA). Supernatants of TGF $\beta 1$ stimulated FBs after gene knockdown and treatment with protease inhibition were collected, centrifuged, and stored at $-20^{\circ} \mathrm{C}$ for further use. Protein levels of human procollagen Ia1 ELISA (R\&D Systems) and human fibronectin ELISA (R\&D Systems) were measured according to the manufacturer's manual. Absorbance was detected by FluoStar Optima microplate reader (BMG Labtech, Ortenberg, Germany). Six-millimeter punch biopsies of healthy skin and hypertrophic scar tissue were lysed in $1 \%$ Triton X-100 lysis buffer (Sigma) and mechanically homogenized using precellyse tissue homogenizer. After centrifugation, lysates were analyzed using DPP4 and urokinase ELISAs (both R\&D Systems) Total protein concentrations were measured using a BCA-kit (Abcam) according to the manufacturer's protocol, and concentrations were normalized to total protein.

Scar planimetry. Collagen bundle alignment has been calculated using Curvealign V4.0 Beta, a curvelet transform-based, open-source MATLAB software. Images of H\&E-stained tissues were edited by Adobe Photoshop CS6 (Adobe Inc, San Jose, CA, USA) to adapt the collagen color, contrast, brightness, and in some cases epidermal alignment to the image border. All images have been processed the same way. Collagen alignment has been calculated according to Curvealign V4.0 Beta manual (August 31, 2017) ${ }^{81}$. Depending on the tissue section, three or four regions of interest per image were selected for calculation. As region size 256 height, 256 width, 1 ROIX, 1 ROIY was chosen. For statistical evaluation, the coefficiency of alignment as comparable value for the relative fiber alignment for every region was calculated. In total, 14 regions of interest calculated from 5 images taken from 4 to 5 animals for each condition.

RNAScope in situ hybridization. FFPE-sections of human skin and scar tissue were prepared according to RNAScope (ACDBio, Bio-Techne, Bristol, UK) pretreatment protocol, hybridized with probes targeting human DPP4 (RNAscope ${ }^{\circledR}$ Probe-Hs-DPP4) and PLAU (RNAscope ${ }^{\circledast}$ Probe-Hs-PLAU), and visualized with 
(A)

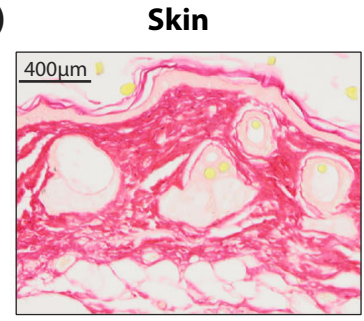

(B)

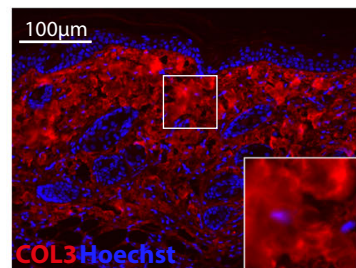

(C)

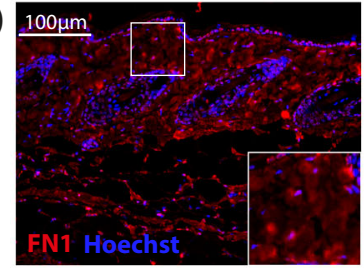

(D)

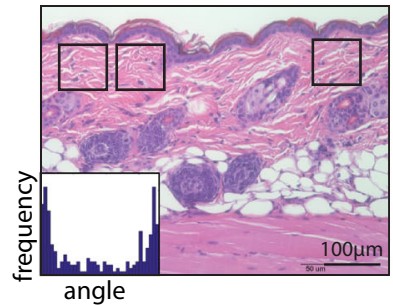

Skin

Scar BC-11

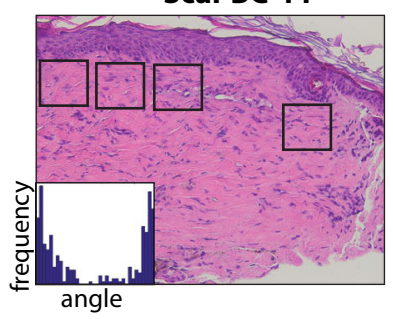

Scar Ctrl
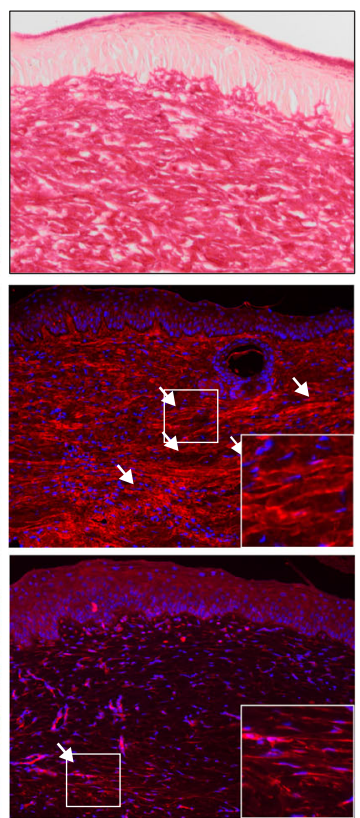

Scar Ctrl

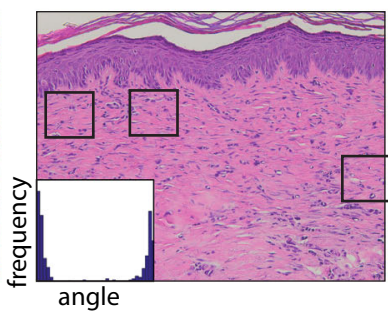

Scar Sitagliptin

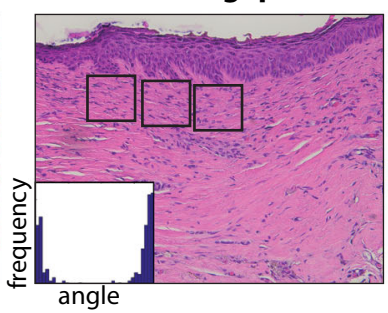

Scar BC-11
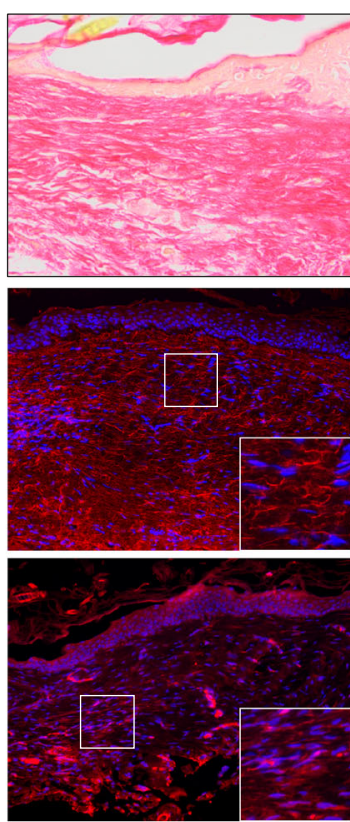

(E)
Scar Sitagliptin
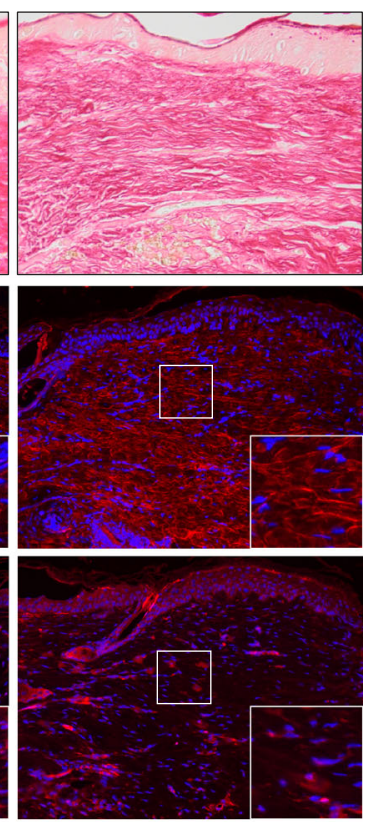

Collagen fiber alignment

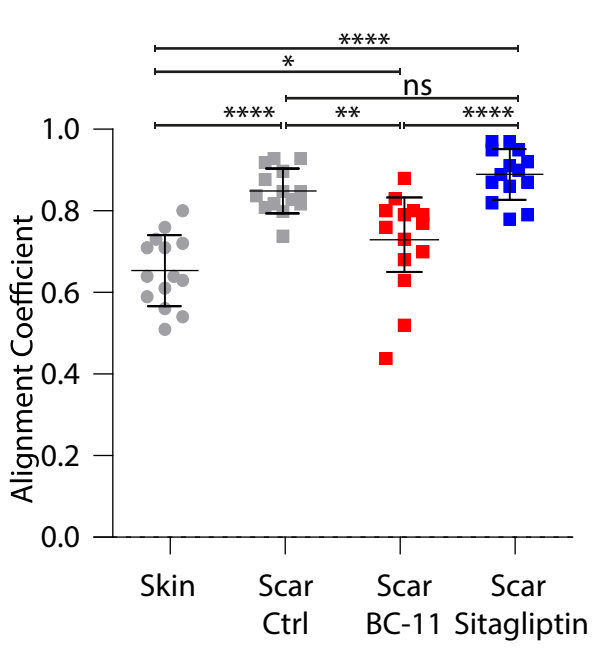

Fig. 9 In vivo application of BC-11 or Sitagliptin improves collagen alignment and fiber orientation in mouse scars. A Picrosirius red staining and immunofluorescent staining of $\mathbf{B}$ Col3a1 and $\mathbf{C}$ fibronectin in mouse skin and scars are shown. Four mice per group were analyzed. Arrows indicate areas of increased matrix density. D H\&E images of mouse skin and scars. Squares indicate areas analyzed for collagen alignment. Histograms illustrate measurement of fiber orientation. E Calculation of alignment coefficient by CurveAlign in mouse skin and scar. $N=4$ mice were analyzed per group, and three to four Regions of interest were calculated per image. Whiskers represent range maximum and minimum values with $<1.5$ interquartile range, boxes represent 25th-75th quartiles, line represents mean. Statistical significance was tested using one-way ANOVA with Tukey post-test. Skin vs Scar untreated $p<0.0001$; Scar untreated vs Scar+BC-11 $p=0.0018$; Scar untreated vs Scar+Sitagliptin $p=0.551$. This mouse experiment was performed once, repetition of the experiment was not permitted by the ethics committee. NS $p>0.05,{ }^{\star} p<0.05,{ }^{\star \star} p<0.01,{ }^{\star \star \star} p<0.001$. Source data are provided as a Source data file.

RNAscope 2.5 HD Assay-RED as suggested by the manufacturer. Images were acquired by AX70 microscope (Olympus, Tokyo, Japan) using the imaging software MetaMorph (Olympus).

Statistical analyses. Two groups with normally distributed data were compared by student's $t$ test. Data of three and more groups were compared by one-way ANOVA with Tukey post hoc test. All statistical analyses were performed in GraphPad Prism v8.0.1 (GraphPad Software, San Diego, USA). $P$-values were marked in figure using asterisks indicating ${ }^{*} p<0.05,{ }^{* *} p<0.01,{ }^{* * *} p<0.001$, $* * * * p<0.0001$.
Reporting summary. Further information on research design is available in the Nature Research Reporting Summary linked to this article.

\section{Data availability}

The scRNAseq data generated in this study have been deposited in the NCBI GEO database under accession "GSE156326". The raw sequencing data are protected and are not available due to data privacy laws. If raw sequencing data are absolutely necessary for replication or extension of our research, they will be made available upon request to the corresponding author within a 2-week timeframe. All other relevant data supporting the key findings of this study are available within the article and its Supplementary 
Information files or from the corresponding author upon reasonable request. Source data are provided with this paper.

Received: 6 August 2020; Accepted: 8 October 2021;

Published online: 29 October 2021

\section{References}

1. Bayat, A., McGrouther, D. A. \& Ferguson, M. W. J. Skin scarring. BMJ: Br. Med. J. 326, 88-92 (2003).

2. Leavitt, T. et al. Scarless wound healing: finding the right cells and signals. Cell Tissue Res. 365, 483-493 (2016).

3. Sen, C. K. et al. Human skin wounds: a major and snowballing threat to public health and the economy. Wound Repair Regen. 17, 763-771 (2009).

4. Bock, O., Schmid-Ott, G., Malewski, P. \& Mrowietz, U. Quality of life of patients with keloid and hypertrophic scarring. Arch. Dermatol. Res. 297, 433 (2006).

5. Van Loey, N. E., Bremer, M., Faber, A. W., Middelkoop, E. \& Nieuwenhuis, M. K. Itching following burns: epidemiology and predictors. Br. J. Dermatol. 158 95-100 (2008)

6. Lee, H. J. \& Jang, Y. J. Recent understandings of biology, prophylaxis and treatment strategies for hypertrophic scars and keloids. Int. J. Mol. Sci. 19, 711 (2018).

7. Hinz, B. Myofibroblasts. Exp. Eye Res. 142, 56-70 (2016).

8. Nabai, L., Pourghadiri, A. \& Ghahary, A. Hypertrophic scarring: current knowledge of predisposing factors, cellular and molecular mechanisms. J. Burn Care Res. 41, 48-56 (2020).

9. Anthonissen, M., Daly, D., Janssens, T. \& Van den Kerckhove, E. The effects of conservative treatments on burn scars: a systematic review. Burns 42, 508-518 (2016).

10. Kafka, M. et al. Evidence of invasive and noninvasive treatment modalities for hypertrophic scars: a systematic review. Wound Repair Regen. 25, 139-144 (2017).

11. Tredget, E. E., Shupp, J. W. \& Schneider, J. C. Scar management following burn injury. J. Burn Care Res. 38, 146-147 (2017).

12. Bao, Y. et al. Comparative efficacy and safety of common therapies in keloids and hypertrophic scars: a systematic review and meta-analysis. Aesthetic Plast. Surg. 44, 207-218 (2020).

13. Kanno, Y. The role of fibrinolytic regulators in vascular dysfunction of systemic sclerosis. Int. J. Mol. Sci. 20, 619 (2019).

14. Menou, A., Duitman, J. \& Crestani, B. The impaired proteases and antiproteases balance in Idiopathic Pulmonary Fibrosis. Matrix Biol. 68-69, 382-403 (2018).

15. Page, M. J. \& Di Cera, E. Serine peptidases: classification, structure and function. Cell. Mol. Life Sci. 65, 1220-1236 (2008).

16. Di Cera, E. Serine proteases. IUBMB Life 61, 510-515 (2009)

17. Rawlings, N. D. \& Barrett, A. J. MEROPS: the peptidase database. Nucleic Acids Res. 27, 325-331 (1999).

18. Gupta, K. K. \& Donahue, D. L. Plasminogen activator inhibitor-1 protects mice against cardiac fibrosis by inhibiting urokinase-type plasminogen activator-mediated plasminogen activation. Sci. Rep. 7, 365 (2017).

19. Schuliga, M. et al. The fibrogenic actions of lung fibroblast-derived urokinase: a potential drug target in IPF. Sci. Rep. 7, 41770 (2017).

20. Makrilakis, K. The role of DPP-4 inhibitors in the treatment algorithm of type 2 diabetes mellitus: when to select, what to expect. Int. J. Environ. Res. Public Health 16, 2720 (2019).

21. Aroor, A. R. et al. Dipeptidyl peptidase-4 (DPP-4) inhibition with linagliptin reduces western diet-induced myocardial TRAF3IP2 expression, inflammation and fibrosis in female mice. Cardiovasc. Diabetol. 16, 61 (2017).

22. Hong, S. K., Choo, E. H., Ihm, S. H., Chang, K. \& Seung, K. B. Dipeptidyl peptidase 4 inhibitor attenuates obesity-induced myocardial fibrosis by inhibiting transforming growth factor-betal and $\operatorname{Smad} 2 / 3$ pathways in high-fat diet-induced obesity rat model. Metab.: Clin. Exp. 76, 42-55 (2017).

23. Kaji, K. et al. Dipeptidyl peptidase- 4 inhibitor attenuates hepatic fibrosis via suppression of activated hepatic stellate cell in rats. J. Gastroenterol. 49, 481-491 (2014).

24. Suzuki, T. et al. Vildagliptin ameliorates pulmonary fibrosis in lipopolysaccharide-induced lung injury by inhibiting endothelial-tomesenchymal transition. Respiratory Res. 18, 177 (2017).

25. Uchida, T. et al. Renoprotective effects of a dipeptidyl peptidase 4 inhibitor in a mouse model of progressive renal fibrosis. Ren. Fail. 39, 340-349 (2017).

26. Schuliga, M., Grainge, C., Westall, G. \& Knight, D. The fibrogenic actions of the coagulant and plasminogen activation systems in pulmonary fibrosis. Int. J. Biochem. Cell Biol. 97, 108-117 (2018)

27. Lay, A. J., Zhang, H. E., McCaughan, G. W. \& Gorrell, M. D. Fibroblast activation protein in liver fibrosis. Front. Biosci. 24, 1-17 (2019).
28. Shi, S., Koya, D. \& Kanasaki, K. Dipeptidyl peptidase-4 and kidney fibrosis in diabetes. Fibrogenes. Tissue Repair 9, 1 (2016)

29. Hu, M. S. \& Longaker, M. T. Dipeptidyl peptidase-4, wound healing, scarring, and fibrosis. Plast. Reconstr. Surg. 138, 1026-1031 (2016).

30. Dong, J. et al. Single-cell RNA-seq analysis unveils a prevalent epithelial/ mesenchymal hybrid state during mouse organogenesis. Genome Biol. 19, 31 (2018).

31. Dekoninck, S. et al. Defining the design principles of skin epidermis postnatal growth. Cell 181, 604-620.e622 (2020).

32. Phan, Q. M. et al. Lef1 expression in fibroblasts maintains developmental potential in adult skin to regenerate wounds. Elife 9, e60066 (2020).

33. Guerrero-Juarez, C. F. et al. Single-cell analysis reveals fibroblast heterogeneity and myeloid-derived adipocyte progenitors in murine skin wounds. Nat. Commun. 10, 650 (2019).

34. Vorstandlechner, V. et al. Deciphering the functional heterogeneity of skin fibroblasts using single-cell RNA sequencing. FASEB J. 34, 3677-3692 (2020).

35. Trapnell, C. et al. The dynamics and regulators of cell fate decisions are revealed by pseudotemporal ordering of single cells. Nat. Biotechnol. 32, 381-386 (2014).

36. Qiu, X. et al. Reversed graph embedding resolves complex single-cell trajectories. Nat. Methods. 14, 979-982 (2017)

37. Kant, S. et al. Duration of scar maturation: retrospective analyses of 361 hypertrophic scars over 5 years. Adv. Ski. Wound Care 32, 26-34 (2019).

38. Ferguson, M. W. \& O'Kane, S. Scar-free healing: from embryonic mechanisms to adult therapeutic intervention. Philos. Trans. R. Soc. Lond. Ser. B, Biol. Sci. 359, 839-850 (2004).

39. Carthy, J. M. TGFbeta signaling and the control of myofibroblast differentiation: implications for chronic inflammatory disorders. J. Cell. Physiol. 233, 98-106 (2018).

40. Roberts, A. B. et al. Transforming growth factor type beta: rapid induction of fibrosis and angiogenesis in vivo and stimulation of collagen formation in vitro. Proc. Natl Acad. Sci. USA 83, 4167-4171 (1986).

41. Lodyga, M. \& Hinz, B. TGF-beta1-A truly transforming growth factor in fibrosis and immunity. Semin. Cell Dev. Biol. 101, 123-39 (2020).

42. Hata, A. \& Chen, Y. G. TGF- $\beta$ signaling from receptors to Smads. Cold Spring Harbor Perspec. Biol. 8, a022061 (2016).

43. Lal, H. et al. Cardiac fibroblast glycogen synthase kinase- $3 \beta$ regulates ventricular remodeling and dysfunction in ischemic heart. Circulation 130 419-430 (2014)

44. Sidgwick, G. P., McGeorge, D. \& Bayat, A. A comprehensive evidence-based review on the role of topicals and dressings in the management of skin scarring. Arch. Dermatol. Res. 307, 461-477 (2015).

45. Muramatsu, T. Midkine, a heparin-binding cytokine with multiple roles in development, repair and diseases. Proc. Jpn. Acad. Ser. B, Phys. Biol. Sci. 86, 410-425 (2010).

46. Miao, L. \& St Clair, D. K. Regulation of superoxide dismutase genes: implications in disease. Free Radic. Biol. Med. 47, 344-356 (2009).

47. Carney, B. C. et al. Reactive oxygen species scavenging potential contributes to hypertrophic scar formation. J. Surg. Res. 244, 312-323 (2019).

48. Toh, P. P. et al. Modulation of metallothionein isoforms is associated with collagen deposition in proliferating keloid fibroblasts in vitro. Exp. Dermatol. 19, 987-993 (2010)

49. Kalekar, L. A. et al. Regulatory T cells in skin are uniquely poised to suppress profibrotic immune responses. Sci. Immunol. 4, eaaw2910 (2019).

50. Vorstandlechner, V. et al. Deciphering the functional heterogeneity of skin fibroblasts using single-cell RNA sequencing. FASEB J. 34, 3677-3692 (2020).

51. Juillerat-Jeanneret, L. Dipeptidyl peptidase IV and its inhibitors: therapeutics for type 2 diabetes and what else? J. Med. Chem. 57, 2197-2212 (2014).

52. Li, Y. et al. Linagliptin inhibits high glucose-induced transdifferentiation of hypertrophic scar-derived fibroblasts to myofibroblasts via IGF/Akt/mTOR signalling pathway. Exp. Dermatol. 28, 19-27 (2019).

53. Suwanai, H., Watanabe, R., Sato, M., Odawara, M. \& Matsumura, H. Dipeptidyl peptidase-4 inhibitor reduces the risk of developing hypertrophic scars and keloids following median sternotomy in diabetic patients: a nationwide retrospective cohort study using the national database of health insurance claims of Japan. Plast. Reconstruc. Surg. 146, 83-89 (2020).

54. Behrendt, N. The urokinase receptor (uPAR) and the uPAR-associated protein (UPARAP/Endo180): membrane proteins engaged in matrix turnover during tissue remodeling. Biol. Chem. 385, 103-136 (2004).

55. Rabieian, R. et al. Plasminogen activator inhibitor type-1 as a regulator of fibrosis. J. Cell Biochem. 119, 17-27 (2018)

56. Wang, D. et al. Sitagliptin ameliorates diabetic nephropathy by blocking TGF 31/Smad signaling pathway. Int. J. Mol. Med. 41, 2784-2792 (2018).

57. Thielitz, A. et al. Inhibitors of dipeptidyl peptidase IV-like activity mediate antifibrotic effects in normal and keloid-derived skin fibroblasts. J. Invest. Dermatol. 128, 855-866 (2008). 
58. Guo, Y. et al. Entanglement of GSK-3 $\beta, \beta$-catenin and TGF- $\beta 1$ signaling network to regulate myocardial fibrosis. J. Mol. Cell. Cardiol. 110, 109-120 (2017).

59. Vallée, A., Lecarpentier, Y., Guillevin, R. \& Vallée, J. N. Interactions between TGF- $\beta 1$, canonical WNT/ $\beta$-catenin pathway and PPAR $\gamma$ in radiationinduced fibrosis. Oncotarget 8, 90579-90604 (2017).

60. Butler, A., Hoffman, P., Smibert, P., Papalexi, E. \& Satija, R. Integrating singlecell transcriptomic data across different conditions, technologies, and species. Nat. Biotechnol. 36, 411 (2018).

61. Mahmoudi, S. et al. Heterogeneity in old fibroblasts is linked to variability in reprogramming and wound healing. Nature 574, 553-558 (2019).

62. Solé-Boldo, L. et al. Single-cell transcriptomes of the human skin reveal agerelated loss of fibroblast priming. Commun. Biol. 3, 188 (2020).

63. Joost, S. et al. The molecular anatomy of mouse skin during hair growth and rest. Cell Stem Cell 26, 441-457.e447 (2020).

64. Ascensión, A. M., Fuertes-Álvarez, S., Ibañez-Solé, O., Izeta, A. \& AraúzoBravo, M. J. Human dermal fibroblast subpopulations are conserved across single-cell RNA sequencing studies. J. Invest. Dermatol. 141, 1735-1744 (2021).

65. Zomer, H. D. \& Trentin, A. G. Skin wound healing in humans and mice: challenges in translational research. J. Dermatol. Sci. 90, 3-12 (2018).

66. Chen, L., Mirza, R., Kwon, Y., DiPietro, L. A. \& Koh, T. J. The murine excisional wound model: contraction revisited. Wound Repair Regen. 23, 874-877 (2015).

67. Cameron, A. M., Adams, D. H., Greenwood, J. E., Anderson, P. J. \& Cowin, A. J. A novel murine model of hypertrophic scarring using subcutaneous infusion of bleomycin. Plast. Reconstruct. Surg. 133, 69-78 (2014).

68. Seo, B. F., Lee, J. Y. \& Jung, S. N. Models of abnormal scarring. BioMed. Res. Int. 2013, 423147 (2013)

69. Griffin, D., Colón, S., Gray, D., Overton, B. \& Wang, B. Design and Development of a Novel Sitagliptin-loaded Transdermal Patch for Diabetes Treatment (2018).

70. Fearmonti, R. M. et al. The modified Patient and Observer Scar Assessment Scale: a novel approach to defining pathologic and nonpathologic scarring. Plast. Reconstr. Surg. 127, 242-247 (2011).

71. Stuart, T. et al. Comprehensive Integration of Single-Cell. Data. Cell. 177, 1888-1902.e21 (2019).

72. Luecken, M. D. \& Theis, F. J. Current best practices in single-cell RNA-seq analysis: a tutorial. Mol. Syst. Biol. 15, e8746 (2019).

73. Rojahn, T. B. et al. Single-cell transcriptomics combined with interstitial fluid proteomics defines cell-type-specific immune regulation in atopic dermatitis. J. Allergy. Clin. Immunol. 146, 1056-1069 (2020).

74. Hammond, T. R. et al. Single-cell RNA sequencing of microglia throughout the mouse lifespan and in the injured brain reveals complex cell-state changes. Immunity 50, 253-271.e256 (2019).

75. Qiu, X. et al. Single-cell mRNA quantification and differential analysis with Census. Nat. Methods 14, 309-315 (2017).

76. Bindea, G. et al. ClueGO: a Cytoscape plug-in to decipher functionally grouped gene ontology and pathway annotation networks. Bioinformatics 25, 1091-1093 (2009).

77. Lotia, S., Montojo, J., Dong, Y., Bader, G. D. \& Pico, A. R. Cytoscape app store. Bioinformatics 29, 1350-1351 (2013).

78. Gschwandtner, M. et al. Histamine suppresses epidermal keratinocyte differentiation and impairs skin barrier function in a human skin model. Allergy 68, 37-47 (2013).

79. Rueden, C. T. et al. ImageJ2: ImageJ for the next generation of scientific image data. BMC Bioinforma. 18, 529 (2017).

80. Mildner, M. et al. Knockdown of filaggrin impairs diffusion barrier function and increases UV sensitivity in a human skin model. J. Investigative Dermatol. 130, 2286-2294 (2010).
81. Liu, Y., Keikhosravi, A., Mehta, G. S., Drifka, C. R. \& Eliceiri, K. W. Methods for quantifying fibrillar collagen alignment. Methods Mol. Biol. 1627, 429-451 (2017).

\section{Acknowledgements}

This research project was financed in part by the FFG Grant "APOSEC" (852748 and 862068; 2015-2019), by the Vienna Business Agency "APOSEC to clinic," (ID 2343727, 2018-2020), and by the Aposcience AG under group leader HJA. MM was funded by the Sparkling Science Program of the Austrian Federal Ministry of Education, Science and Research (SPA06/055). We thank the HPH Haselsteiner and the CRISCAR Familienstiftung for their belief in this private public partnership to augment basic and translational clinical research. We thank Stefan Spalt for his support. The authors acknowledge the core facilities of the Medical University of Vienna, a member of Vienna Life Science Instruments.

\section{Author contributions}

M.M., H.J.A., E.T. and V.V. provided study conception and design; W.H. and C.R. provided patient sample material; H.J.A. and M.M. acquired funding; V.V., D.C., K.K., M.D., Y.C. and B.G. conducted experiments and prepared samples; V.V., M.L. and M.M performed data analysis, visualization and figure design; V.V., M.L., K.K., E.T., K.H. and M.M. participated in data interpretation; V.V., M.L. and M.M. drafted the manuscript. All authors reviewed the manuscript.

\section{Competing interests}

The authors declare no competing interests.

\section{Additional information}

Supplementary information The online version contains supplementary material available at https://doi.org/10.1038/s41467-021-26495-2.

Correspondence and requests for materials should be addressed to Hendrik Jan Ankersmit or Michael Mildner.

Peer review information Nature Communications thanks the anonymous reviewer(s) for their contribution to the peer review of this work. Peer reviewer reports are available.

Reprints and permission information is available at http://www.nature.com/reprints

Publisher's note Springer Nature remains neutral with regard to jurisdictional claims in published maps and institutional affiliations.

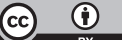

Open Access This article is licensed under a Creative Commons Attribution 4.0 International License, which permits use, sharing, adaptation, distribution and reproduction in any medium or format, as long as you give appropriate credit to the original author(s) and the source, provide a link to the Creative Commons license, and indicate if changes were made. The images or other third party material in this article are included in the article's Creative Commons license, unless indicated otherwise in a credit line to the material. If material is not included in the article's Creative Commons license and your intended use is not permitted by statutory regulation or exceeds the permitted use, you will need to obtain permission directly from the copyright holder. To view a copy of this license, visit http://creativecommons.org/ licenses/by/4.0\%

(C) The Author(s) 2021 